Supporting Information for:

\title{
Using Redox-Active Ligands to Generate Actinide Ligand Radical Species
}

Shane S. Galley, ${ }^{a} \uparrow$ Scott A. Pattenaude, ${ }^{a} \dagger$ Debmalya Ray ${ }_{,}^{f}$ Carlo Alberto Gaggioli, ${ }^{b}$ Megan A.

Whitefoot ${ }^{a}$ Yusen Qiao, ${ }^{c}$ Robert F. Higgins, ${ }^{c}$ W.L. Nelson, ${ }^{d}$ Ryan Baumbach ${ }^{d}{ }^{d}$ Joseph M.

Sperling, ${ }^{e}$ Matthias Zeller, ${ }^{a}$ Tyler S. Collins,${ }^{a}$ Eric J. Schelter ${ }^{d}$ Laura Gagliardi, ${ }^{b}$ Thomas E. Albrecht-Schönzart, ${ }^{e}$ Suzanne C. Bart ${ }^{a *}$

${ }^{a}$ H.C. Brown Laboratory, Department of Chemistry, Purdue University, West Lafayette, Indiana 47907, United States

bDepartment of Chemistry, Pritzker School of Molecular Engineering, James Franck Institute, and Chicago Center for Theoretical Chemistry, The University of Chicago, 5735 S Ellis Ave, Chicago, IL 60637, United States

'P. Roy and Diana T. Vagelos Laboratories, Department of Chemistry, University of Pennsylvania, Philadelphia, Pennsylvania, 19104, United States

${ }^{d}$ National High Magnetic Field Laboratory, Florida State University, Tallahassee, Florida, 32310, United States; Department of Physics, Florida State University, Tallahassee, Florida, 32306, United States

${ }^{\mathrm{e}}$ Department of Chemistry and Biochemistry, Florida State University, Tallahassee, Florida 32306, United States

${ }^{\mathrm{f}}$ Department of Chemistry, Supercomputing Institute, and Chemical Theory Centre, University of Minnesota, Minneapolis, Minnesota, 55455, United States

*To whom all correspondence should be addressed: sbart@purdue.edu 


\section{Table of Contents}

\section{Characterization and Analysis}

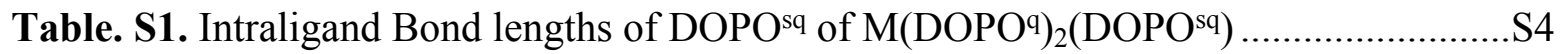

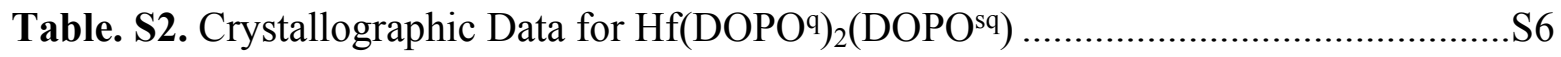

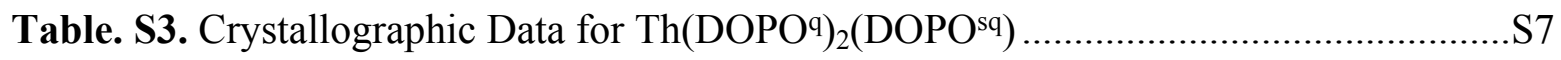

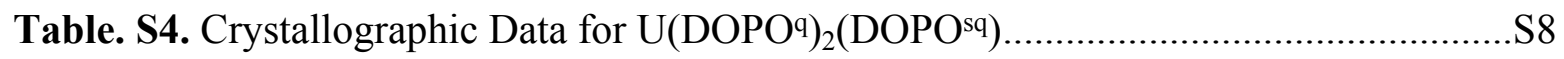

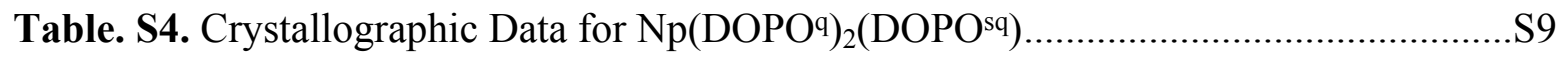

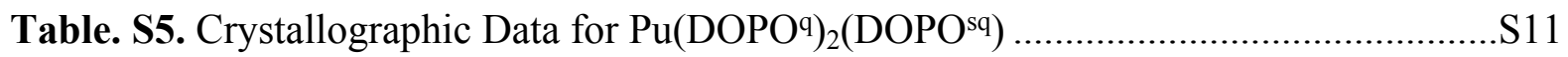

Table S6. Metrical Oxidation States for Ligands in $\mathrm{Pu}\left(\mathrm{DOPO}^{\mathrm{q}}\right)_{2}\left(\mathrm{DOPO}^{\mathrm{sq}}\right)$......................S 12

Table S7. Metrical Oxidation States for Ligands in $\mathrm{Np}\left(\mathrm{DOPO}^{\mathrm{q}}\right)_{2}\left(\mathrm{DOPO}^{\mathrm{sq}}\right) \ldots \ldots \ldots \ldots \ldots \ldots \ldots . . . . . . . . . . . . .212$

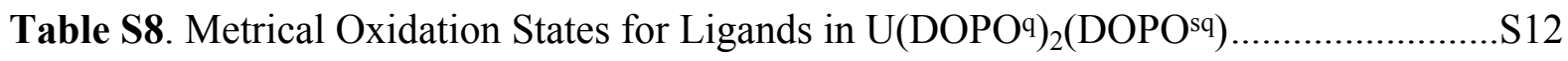

Table S9. Metrical Oxidation States for Ligands in $\mathrm{Th}\left(\mathrm{DOPO}^{\mathrm{q}}\right)_{2}\left(\mathrm{DOPO}^{\mathrm{sq}}\right)$......................S13

Table S10. Metrical Oxidation States for Ligands in $\mathrm{Hf}\left(\mathrm{DOPO}^{\mathrm{q}}\right)_{2}\left(\mathrm{DOPO}^{\mathrm{sq}}\right)$...................S 13

Figure S1. Solution UV-Vis-NIR spectrum of U(DOPO $)_{2}\left(\mathrm{DOPO}^{\mathrm{sq}}\right)$ in THF at $25^{\circ} \mathrm{C}$. Solvent

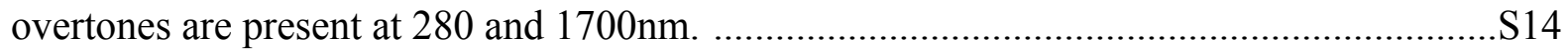

Figure S2. Solution UV-Vis-NIR spectrum of $\mathrm{M}\left(\mathrm{DOPO}^{\mathrm{q}}\right)_{2}\left(\mathrm{DOPO}^{\mathrm{sq}}\right)(\mathrm{M}: \mathrm{Hf}, \mathrm{Th})$ in THF at $25^{\circ} \mathrm{C}$. Solvent overtones are present at $280 \mathrm{~nm}$.

Figure S3. ${ }^{1} \mathrm{H}$ NMR spectrum of $\mathrm{Hf}\left(\mathrm{DOPO}^{\mathrm{q}}\right)_{2}\left(\mathrm{DOPO}^{\mathrm{sq}}\right)$ in $\mathrm{C}_{6} \mathrm{D}_{6}$ at $25^{\circ} \mathrm{C} \ldots \ldots \ldots \ldots \ldots \ldots \ldots . . . . . . . . . . . . . .616$

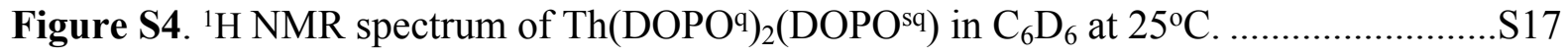

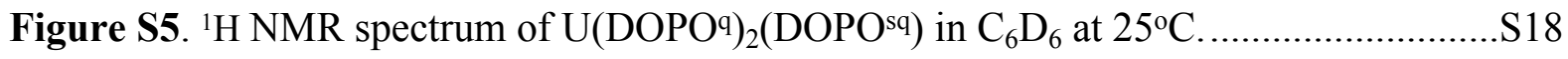

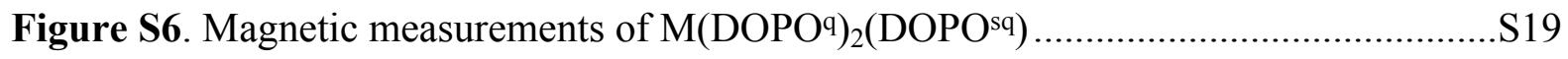

Table S11. Spin states absolute and relative energies for $\mathrm{M}\left(\mathrm{DOPO}^{\mathrm{q}}\right)_{2}\left(\mathrm{DOPO}^{\mathrm{sq}}\right)\left(\mathrm{Hartree}\right.$ and $\mathrm{cm}^{-}$

${ }^{1}$ respectively) (at the CASSCF and CASPT2 level)......................................................S20

Table S12. Mulliken spin density and LoProp charge on metals and radical $\mathrm{N}$ for $\mathrm{M}\left(\mathrm{DOPO}^{\mathrm{q}}\right)_{2}\left(\mathrm{DOPO}^{\mathrm{sq}}\right)$ complexes.

Table S13: Dominant electronic configurations of each spin states for M-DOPO complexes in the CASSCF level......................................................... S21 
Figure S7. Active space orbitals added to the $\operatorname{CASSCF}(7,12)$ for the quartet spin state for $\mathrm{U}\left(\mathrm{DOPO}^{\mathrm{q}}\right)_{2}\left(\mathrm{DOPO}^{\mathrm{sq}}\right)$ (occupation numbers in brackets).

Figure S8. Active space orbitals added to the $\operatorname{CASSCF}(7,12)$ for the doublet spin state for $\mathrm{U}\left(\mathrm{DOPO}^{\mathrm{q}}\right)_{2}\left(\mathrm{DOPO}^{\mathrm{sq}}\right)$ (occupation numbers in brackets).

Figure S9. Active space orbitals added to the $\operatorname{CASSCF}(8,12)$ for the triplet spin state for $\mathrm{Np}\left(\text { DOPO }^{q}\right)_{2}\left(\right.$ DOPO $\left.^{\mathrm{sq}}\right)$ (occupation numbers in brackets).

Figure S10. Active space orbitals added to the $\operatorname{CASSCF}(9,12)$ for the sextet spin state for $\mathrm{Pu}\left(\mathrm{DOPO}^{\mathrm{q}}\right)_{2}\left(\mathrm{DOPO}^{\mathrm{sq}}\right)$ (occupation numbers in brackets)..............................................S2

Figure S11. Active space orbitals added to the $\operatorname{CASSCF}(9,12)$ for the quartet spin state for $\mathrm{Pu}\left(\mathrm{DOPO}^{\mathrm{q}}\right)_{2}\left(\mathrm{DOPO}^{\mathrm{sq}}\right)$ (occupation numbers in brackets).

Figure S12. Active space orbitals added to the $\operatorname{CASSCF}(5,5)$ for the doublet spin state for $\mathrm{Th}\left(\mathrm{DOPO}^{\mathrm{q}}\right)_{2}\left(\mathrm{DOPO}^{\mathrm{sq}}\right)$ (occupation numbers in brackets).

Figure S13. Active space orbitals added to the $\operatorname{CASSCF}(5,5)$ for the doublet spin state for $\mathrm{Hf}\left(\mathrm{DOPO}^{\mathrm{q}}\right)_{2}\left(\mathrm{DOPO}^{\mathrm{sq}}\right)$ (occupation numbers in brackets)............................................S28

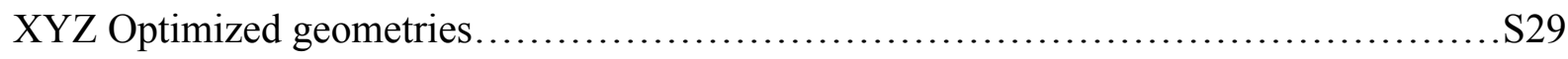

References................................................................ 34 


\begin{tabular}{|c|c|c|c|c|c|}
\hline \multicolumn{6}{|c|}{$\begin{array}{c}\text { Table S1: Intraligand Bond Lengths }(\AA) \text { of } \mathrm{DOPO}^{\mathrm{sq}} \text { of } \mathrm{M}\left(\mathrm{DOPO}^{\mathrm{q}}\right)_{2}\left(\mathrm{DOPO}^{\mathrm{sq}}\right) \mathrm{M}: \mathrm{Hf}, \mathrm{Th}, \mathrm{U} \\
\mathrm{Np}, \mathrm{Pu}\end{array}$} \\
\hline & Hf & $\mathrm{Th}$ & $\mathrm{U}$ & $\mathrm{Np}$ & $\mathrm{Pu}$ \\
\hline O1-C1 & $1.318(5)$ & $1.317(5)$ & $1.325(7)$ & $1.324(9)$ & $1.317(3)$ \\
\hline O3-C7 & $1.308(5)$ & $1.323(5)$ & $1.347(7)$ & $1.348(9)$ & $1.324(3)$ \\
\hline N1-C6 & $1.343(5)$ & $1.352(5)$ & $1.340(8)$ & $1.368(10)$ & $1.345(3)$ \\
\hline N1-C12 & $1.340(5)$ & $1.357(5)$ & $1.348(8)$ & $1.373(9)$ & $1.346(3)$ \\
\hline $\mathrm{C} 1-\mathrm{C} 2$ & $1.416(6)$ & $1.415(5)$ & $1.411(9)$ & $1.417(11)$ & $1.404(3)$ \\
\hline C1-C6 & $1.417(6)$ & $1.430(5)$ & $1.411(9)$ & $1.436(11)$ & $1.426(3)$ \\
\hline C2-C3 & $1.388(7)$ & $1.396(6)$ & $1.384(9)$ & $1.396(11)$ & $1.392(4)$ \\
\hline C3-C4 & $1.419(7)$ & $1.419(5)$ & $1.432(9)$ & $1.447(11)$ & $1.416(4)$ \\
\hline C4-C5 & $1.397(6)$ & $1.382(5)$ & $1.388(9)$ & $1.358(11)$ & $1.383(3)$ \\
\hline C5-C6 & $1.395(6)$ & $1.401(6)$ & $1.406(9)$ & $1.363(11)$ & $1.401(3)$ \\
\hline C7-C8 & $1.418(6)$ & $1.402(5)$ & $1.406(9)$ & $1.406(10)$ & $1.399(3)$ \\
\hline C7-C12 & $1.410(6)$ & $1.426(5)$ & $1.407(9)$ & $1.402(11)$ & $1.423(3)$ \\
\hline C8-C9 & $1.389(6)$ & $1.400(6)$ & $1.396(9)$ & $1.389(12)$ & $1.392(3)$ \\
\hline C9-C10 & $1.429(6)$ & $1.398(5)$ & $1.405(9)$ & $1.418(12)$ & $1.411(3)$ \\
\hline C10-C11 & $1.405(5)$ & $1.402(6)$ & $1.387(9)$ & $1.380(10)$ & $1.383(4)$ \\
\hline C11-C12 & $1.402(6)$ & $1.396(6)$ & $1.403(9)$ & $1.407(11)$ & $1.401(3)$ \\
\hline
\end{tabular}




\section{Single Crystal X-Ray Structure Determination:}

Data were collected either on a Nonius Kappa CCD diffractometer with a molybdenum wavelength

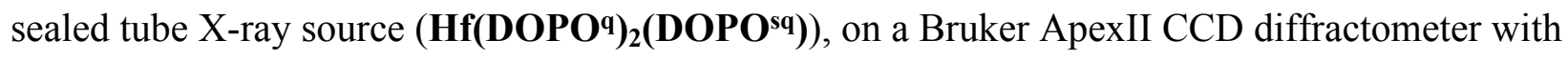
a copper microscource $\left(\mathbf{U}_{\left(\mathbf{D O P O}^{q}\right.}\right)_{2}\left(\mathbf{D O P O}^{\mathrm{sq}}\right)$ ) or a molybdenum microsource $\left(\mathrm{Mo} \mathrm{K}_{\alpha}, \lambda=\right.$

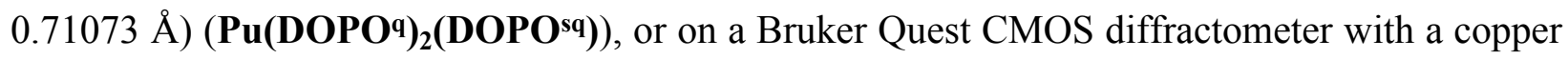
microsource $\left(\mathrm{Cu} \mathrm{K}_{\alpha}, \lambda=1.54178 \AA\right)\left(\mathbf{T h}\left(\mathbf{D O P O}^{\mathrm{q}}\right)_{2}\left(\mathbf{D O P O}^{\mathrm{sa}}\right)\right)$. Data from the Nonius KappaCCD instrument were collected using the Nonius Collect software ${ }^{1}$ and the data were processed using HKL $3000^{2}$ and data were corrected for absorption and scaled using Scalepack. ${ }^{2}$ Data from the Bruker instruments were collected using the Apex 2 or Apex 3 software. The space groups were assigned using XPREP from the Shelxtl suite of programs ${ }^{3}$ and the structures were solved by direct methods with SHELXS ${ }^{4}$ and refined by full matrix least squares against $\mathrm{F}^{2}$ with all reflections using the graphical user interface ShelXle ${ }^{5}$ for refinement program SHELXL. ${ }^{6} \mathrm{H}$ atoms attached to carbon and boron atoms were positioned geometrically and constrained to ride on their parent atoms, with carbon hydrogen bond distances of $0.95 \AA$ for and aromatic C-H, 1.00, 0.99 and 0.98 $\AA$ for aliphatic $\mathrm{C}-\mathrm{H}, \mathrm{CH}_{2}$ and $\mathrm{CH}_{3}$ moieties, respectively. Methyl $\mathrm{H}$ atoms were allowed to rotate but not to tip to best fit the experimental electron density. $\mathrm{U}_{\text {iso }}(\mathrm{H})$ values were set to a multiple of $\mathrm{U}_{\text {eq }}(\mathrm{C} / \mathrm{B})$ with 1.5 for $\mathrm{CH}_{3}$, and 1.2 for $\mathrm{C}-\mathrm{H}$ and $\mathrm{CH}_{2}$. 
Table S2. Crystallographic Data for $\mathrm{Hf}(\mathrm{DOPO})_{2}\left(\mathrm{DOPO}^{\mathrm{sq}}\right)$.

\begin{tabular}{|c|c|}
\hline Chemical formula & $2\left(\mathrm{C}_{84} \mathrm{H}_{114} \mathrm{HfN}_{3} \mathrm{O}_{9}\right) \cdot 3\left(\mathrm{C}_{5} \mathrm{H}_{12}\right)$ \\
\hline$M_{\mathrm{r}}$ & 3193.00 \\
\hline Crystal system, space group & Monoclinic, $P 2_{1} / n$ \\
\hline Temperature $(\mathrm{K})$ & 100 \\
\hline$a, b, c(\AA)$ & $14.8170(3), 24.3245(5), 24.5275(5)$ \\
\hline$\beta\left({ }^{\circ}\right)$ & $97.6363(10)$ \\
\hline$V\left(\AA^{3}\right)$ & $8761.7(3)$ \\
\hline$Z$ & 2 \\
\hline Radiation type & Mo $K \alpha$ \\
\hline$\mu\left(\mathrm{mm}^{-1}\right)$ & 1.25 \\
\hline Crystal size $(\mathrm{mm})$ & $0.28 \times 0.27 \times 0.14$ \\
\hline \multicolumn{2}{|l|}{ Data collection } \\
\hline Diffractometer & Nonius Kappa CCD diffractometer \\
\hline Absorption correction & Multi-scan, SCALEPACK (Otwinowski \& Minor, 1997) \\
\hline$T_{\min }, T_{\max }$ & $0.467,0.845$ \\
\hline $\begin{array}{l}\text { No. of measured, independent and observed } \\
{[I>2 \sigma(I)] \text { reflections }}\end{array}$ & $63438,20969,12512$ \\
\hline$R_{\text {int }}$ & 0.075 \\
\hline$(\sin \theta / \lambda)_{\max }\left(\AA^{-1}\right)$ & 0.693 \\
\hline \multicolumn{2}{|l|}{ Refinement } \\
\hline$R\left[F^{2}>2 \sigma\left(F^{2}\right)\right], w R\left(F^{2}\right), S$ & $0.052,0.111,1.05$ \\
\hline No. of reflections & 20969 \\
\hline No. of parameters & 1004 \\
\hline No. of restraints & 31 \\
\hline \multirow[t]{2}{*}{ H-atom treatment } & $\mathrm{H}$-atom parameters constrained \\
\hline & $\begin{array}{l}w=1 /\left[\sigma^{2}\left(F_{\mathrm{o}}^{2}\right)+(0.0173 P)^{2}+24.031 P\right] \\
\text { where } P=\left(F_{\mathrm{o}}^{2}+2 F_{\mathrm{c}}^{2}\right) / 3\end{array}$ \\
\hline$\Delta \rho_{\max }, \Delta \rho_{\min }\left(\mathrm{e} \AA^{-3}\right)$ & $2.05,-2.44$ \\
\hline
\end{tabular}

Computer programs: Nonius Collect (Nonius, 1998), HKL-3000 (Otwinowski \& Minor, 1997), SHELXS97 (Sheldrick, 2008), SHELXL2014/7 (Sheldrick, 2014), SHELXLE Rev714 (Hübschle et al., 2011).

Refinement notes: A pentane molecule is disordered around a crystallographic inversion center. Its geometry was restrained to be similar to that of another not disordered pentane molecule, and the $U^{\mathrm{ij}}$ components of ADPs were restrained to be similar for atoms closer than $2.0 \AA$. 
Table S3. Crystallographic Data for $\mathrm{Th}\left(\mathrm{DOPO}^{\mathrm{q}}\right)_{2}\left(\mathrm{DOPO}^{\mathrm{sq}}\right)$.

\begin{tabular}{|c|c|}
\hline Chemical formula & $\mathrm{C}_{84} \mathrm{H}_{114} \mathrm{~N}_{3} \mathrm{O}_{9} \mathrm{Th} \cdot 4\left(\mathrm{C}_{5} \mathrm{H}_{5} \mathrm{~N}\right)$ \\
\hline$M_{\mathrm{r}}$ & 1858.21 \\
\hline Crystal system, space group & Monoclinic, $P 2_{1} / n$ \\
\hline Temperature (K) & 100 \\
\hline$a, b, c(\AA)$ & $14.3886(3), 26.2951(5), 25.7121(5)$ \\
\hline$\beta\left(^{\circ}\right)$ & $100.7004(10)$ \\
\hline$V\left(\AA^{3}\right)$ & $9559.0(3)$ \\
\hline$Z$ & 4 \\
\hline Radiation type & $\mathrm{Cu} K \alpha$ \\
\hline$\mu\left(\mathrm{mm}^{-1}\right)$ & 5.50 \\
\hline Crystal size $(\mathrm{mm})$ & $0.50 \times 0.30 \times 0.25$ \\
\hline \multicolumn{2}{|l|}{ Data collection } \\
\hline Diffractometer & Bruker AXS D8 Quest CMOS diffractometer \\
\hline Absorption correction & $\begin{array}{l}\text { Multi-scan, SADABS 2016/2: Krause, L., Herbst-Irmer, R., Sheldrick } \\
\text { G.M. \& Stalke D., J. Appl. Cryst. } 48 \text { (2015) 3-10 }\end{array}$ \\
\hline$T_{\min }, T_{\max }$ & $0.616,0.754$ \\
\hline $\begin{array}{l}\text { No. of measured, independent and } \\
\text { observed }[I>2 \sigma(I)] \text { reflections }\end{array}$ & $50836,19494,13646$ \\
\hline$R_{\text {int }}$ & 0.054 \\
\hline$(\sin \theta / \lambda)_{\max }\left(\AA^{-1}\right)$ & 0.639 \\
\hline \multicolumn{2}{|l|}{ Refinement } \\
\hline$R\left[F^{2}>2 \sigma\left(F^{2}\right)\right], w R\left(F^{2}\right), S$ & $0.045,0.110,1.03$ \\
\hline No. of reflections & 19494 \\
\hline No. of parameters & 1126 \\
\hline \multirow[t]{2}{*}{ H-atom treatment } & H-atom parameters constrained \\
\hline & $w=1 /\left[\sigma^{2}\left(F_{\mathrm{o}}^{2}\right)+(0.0446 P)^{2}+11.0647 P\right]$ where $P=\left(F_{\mathrm{o}}^{2}+2 F_{\mathrm{c}}^{2}\right) / 3$ \\
\hline$\Delta \rho_{\max }, \Delta \rho_{\min }\left(\mathrm{e} \AA^{-3}\right)$ & $3.08,-0.82$ \\
\hline
\end{tabular}

Computer programs: Apex3 v2016.9-0 (Bruker, 2016), SAINT V8.37A (Bruker, 2016), SHELXS97

(Sheldrick, 2008), SHELXL2016/6 (Sheldrick, 2015, 2016), SHELXLE Rev714 (Hübschle et al., 2011). 
Table S4. Crystallographic Data for U(DOPO $)_{2}\left(\mathrm{DOPO}^{\mathrm{sq}}\right)$.

\begin{tabular}{|c|c|}
\hline Chemical formula & $\mathrm{C}_{84} \mathrm{H}_{114} \mathrm{~N}_{3} \mathrm{O}_{9} \mathrm{U} \cdot 2\left(\mathrm{C}_{5} \mathrm{H}_{5} \mathrm{~N}\right)$ \\
\hline$M_{\mathrm{r}}$ & 1706.00 \\
\hline Crystal system, space group & Monoclinic, $P 2_{1} / c$ \\
\hline Temperature (K) & 128 \\
\hline$a, b, c(\AA)$ & $14.940(2), 15.505(3), 37.606(6)$ \\
\hline$\beta\left({ }^{\circ}\right)$ & $91.263(3)$ \\
\hline$V\left(\AA^{3}\right)$ & $8709(2)$ \\
\hline$Z$ & 4 \\
\hline Radiation type & Mo $K \alpha$ \\
\hline$\mu\left(\mathrm{mm}^{-1}\right)$ & 1.92 \\
\hline Crystal size $(\mathrm{mm})$ & $0.06 \times 0.05 \times 0.04$ \\
\hline \multicolumn{2}{|l|}{ Data collection } \\
\hline Diffractometer & Bruker ApexII CCD diffractometer \\
\hline Absorption correction & $\begin{array}{l}\text { Multi-scan, SADABS 2014/2: Krause, L., Herbst-Irmer, R., Sheldrick } \\
\text { G.M. \& Stalke D., J. Appl. Cryst. } 48 \text { (2015) 3-10 }\end{array}$ \\
\hline$T_{\min }, T_{\max }$ & $0.413,0.681$ \\
\hline $\begin{array}{l}\text { No. of measured, independent and observed } \\
{[I>2 \sigma(I)] \text { reflections }}\end{array}$ & $110574,19776,11429$ \\
\hline$R_{\text {int }}$ & 0.204 \\
\hline$(\sin \theta / \lambda)_{\max }\left(\AA^{-1}\right)$ & 0.648 \\
\hline \multicolumn{2}{|l|}{ Refinement } \\
\hline$R\left[F^{2}>2 \sigma\left(F^{2}\right)\right], w R\left(F^{2}\right), S$ & $0.068,0.169,1.04$ \\
\hline No. of reflections & 19776 \\
\hline No. of parameters & 1019 \\
\hline H-atom treatment & H-atom parameters constrained \\
\hline$\Delta \rho_{\max }, \Delta \rho_{\min }\left(\mathrm{e} \AA^{-3}\right)$ & $2.17,-3.04$ \\
\hline
\end{tabular}

Computer programs: Apex2 (Bruker, 2016), SAINT V8.37A (Bruker, 2016), SHELXS97 (Sheldrick, 2008), SHELXL2016/6 (Sheldrick, 2015, 2016), SHELXLE Rev714 (Hübschle et al., 2011). 
Table S5. Crystallographic Data for $\mathrm{Np}\left(\mathrm{DOPO}^{\mathrm{q}}\right)_{2}\left(\mathrm{DOPO}^{\mathrm{sq}}\right)$.

\begin{tabular}{|c|c|}
\hline Chemical formula & $\mathrm{C}_{84} \mathrm{H}_{114} \mathrm{~N}_{3} \mathrm{NpO}_{9} \cdot 1.766\left(\mathrm{C}_{5} \mathrm{H}_{5} \mathrm{~N}\right)$ \\
\hline$M_{\mathrm{r}}$ & 1686.40 \\
\hline Crystal system, space group & Monoclinic, $P 2_{1} / c$ \\
\hline Temperature $(\mathrm{K})$ & 130 \\
\hline$a, b, c(\AA)$ & $14.596(3), 15.571(3), 38.085(7)$ \\
\hline$\beta\left(^{\circ}\right)$ & $91.074(3)$ \\
\hline$V\left(\AA^{3}\right)$ & $8655(3)$ \\
\hline$Z$ & 4 \\
\hline Radiation type & Mo $K \alpha$ \\
\hline$\mu\left(\mathrm{mm}^{-1}\right)$ & 1.26 \\
\hline Crystal size $(\mathrm{mm})$ & $0.06 \times 0.05 \times 0.04$ \\
\hline \multicolumn{2}{|l|}{ Data collection } \\
\hline Diffractometer & $\begin{array}{l}\text { Bruker ApexII CCD } \\
\text { diffractometer }\end{array}$ \\
\hline Absorption correction & $\begin{array}{l}\text { Multi-scan } \\
\text { SADABS 2016/2: Krause, L., Herbst-Irmer, R., } \\
\text { Sheldrick G.M. \& Stalke D., J. Appl. Cryst. } 48 \\
\text { (2015) 3-10 }\end{array}$ \\
\hline$T_{\min }, T_{\max }$ & $0.499,0.949$ \\
\hline $\begin{array}{l}\text { No. of measured, independent and } \\
\text { observed }[I>2 \sigma(I)] \text { reflections }\end{array}$ & $98469,19631,11737$ \\
\hline$R_{\text {int }}$ & 0.167 \\
\hline$(\sin \theta / \lambda)_{\max }\left(\AA^{-1}\right)$ & 0.649 \\
\hline \multicolumn{2}{|l|}{ Refinement } \\
\hline$R\left[F^{2}>2 \sigma\left(F^{2}\right)\right], w R\left(F^{2}\right), S$ & $0.085,0.198,1.16$ \\
\hline No. of reflections & 19631 \\
\hline No. of parameters & 1122 \\
\hline No. of restraints & 492 \\
\hline \multirow[t]{2}{*}{ H-atom treatment } & H-atom parameters constrained \\
\hline & $\begin{array}{l}w=1 /\left[\sigma^{2}\left(F_{\mathrm{o}}^{2}\right)+26.0841 P\right] \\
\text { where } P=\left(F_{\mathrm{o}}^{2}+2 F_{\mathrm{c}}^{2}\right) / 3\end{array}$ \\
\hline$\left.\Delta\rangle_{\max }, \Delta\right\rangle_{\min }\left(\mathrm{e} \AA^{-3}\right)$ & $1.90,-2.20$ \\
\hline
\end{tabular}

Computer programs: Apex2 (Bruker, 2016), SAINT V8.37A (Bruker, 2016), SHELXS97 (Sheldrick, 2008), SHELXL2018/3 (Sheldrick, 2018), SHELXLE Rev714 (Hübschle et al., 2011). 
Refinement Notes: A tert-butyl group is rotationally disordered. The two disordered moieties were

\begin{tabular}{|c|c|}
\hline Chemical formula & $\mathrm{C}_{84} \mathrm{H}_{114} \mathrm{~N}_{3} \mathrm{O}_{9} \mathrm{Pu} \cdot 2\left(\mathrm{C}_{5} \mathrm{H}_{5} \mathrm{~N}\right)$ \\
\hline$M_{\mathrm{r}}$ & 1709.99 \\
\hline Crystal system, space group & Monoclinic, $P 2_{1} / c$ \\
\hline Temperature $(\mathrm{K})$ & 130 \\
\hline$a, b, c(\AA)$ & $14.9354(8), 15.5118(8), 37.7368(19)$ \\
\hline$\beta\left(^{\circ}\right)$ & $90.9134(12)$ \\
\hline$V\left(\AA^{3}\right)$ & $8741.6(8)$ \\
\hline$Z$ & 4 \\
\hline Radiation type & Mo $K \alpha$ \\
\hline$\mu\left(\mathrm{mm}^{-1}\right)$ & 0.81 \\
\hline Crystal size (mm) & $0.06 \times 0.05 \times 0.04$ \\
\hline \multicolumn{2}{|l|}{ Data collection } \\
\hline Diffractometer & Bruker ApexII CCD diffractometer \\
\hline Absorption correction & $\begin{array}{l}\text { Multi-scan, SADABS 2014/2: Krause, L., Herbst-Irmer, R., Sheldrick } \\
\text { G.M. \& Stalke D., J. Appl. Cryst. } 48 \text { (2015) 3-10 }\end{array}$ \\
\hline$T_{\min }, T_{\max }$ & $0.704,0.746$ \\
\hline $\begin{array}{l}\text { No. of measured, independent and observed } \\
{[I>2 \sigma(I)] \text { reflections }}\end{array}$ & $133566,20023,15750$ \\
\hline$R_{\text {int }}$ & 0.063 \\
\hline$(\sin \theta / \lambda)_{\max }\left(\AA^{-1}\right)$ & 0.650 \\
\hline \multicolumn{2}{|l|}{ Refinement } \\
\hline$R\left[F^{2}>2 \sigma\left(F^{2}\right)\right], w R\left(F^{2}\right), S$ & $0.030,0.064,1.03$ \\
\hline No. of reflections & 20023 \\
\hline No. of parameters & 1018 \\
\hline
\end{tabular}

restrained to have similar geometries. Uij components of ADPs for disordered atoms closer to each other than 2.0 Angstrom were restrained to be similar. Subject to these conditions the occupancy ratio refined to $0.489(9)$ to $0.511(9)$.

Two pyridyl solvate molecules were refined as disordered. One positionally by a slight rotation in place. The other is disordered around an inversion center, with either two molecules side by side (major moiety), or just one molecule split over two mutually exclusive symmetry equivalent positions.

All pyridyl rings were constrained to resemble ideal hexagons (AFIX 66 command). Uij components of ADPs for disordered atoms closer to each other than 2.0 Angstrom were restrained to be similar. Subject to these conditions the occupancy ratio for the first molecule refined to $0.553(18)$ to $0.447(18)$. That for the second molecule to $0.531(10)$ to $0.235(5)$. 


\begin{tabular}{|l|l|}
\hline H-atom treatment & H-atom parameters constrained \\
\hline$\Delta \rho_{\max }, \Delta \rho_{\min }\left(\mathrm{e} \AA^{-3}\right)$ & $0.91,-0.46$ \\
\hline
\end{tabular}

Table S5. Crystallographic Data for $\mathrm{Pu}\left(\mathrm{DOPO}^{\mathrm{q}}\right)_{2}\left(\mathrm{DOPO}^{\mathrm{sq}}\right)$.

Computer programs: Apex2 (Bruker, 2016), SAINT V8.37A (Bruker, 2016), SHELXS97 (Sheldrick, 2008), SHELXL2016/6 (Sheldrick, 2015, 2016), SHELXLE Rev714 (Hübschle et al., 2011). 
Table S6. Metrical Oxidation States for Ligands in $\mathrm{Pu}\left(\mathrm{DOPO}^{\mathrm{q}}\right)_{2}\left(\mathrm{DOPO}^{\mathrm{sq}}\right)$.

\begin{tabular}{|c|c|c|c|c|c|c|c|c|c|}
\hline & C1-N & C2-O & C1-C2 & C2-C3 & C3-C4 & C4-C5 & C5-C6 & C1-C6 & MOS Total \\
\hline & 1.345 & 1.316 & 1.423 & 1.404 & 1.392 & 1.416 & 1.384 & 1.401 & -2.008 \\
\hline & 1.346 & 1.324 & 1.423 & 1.398 & 1.392 & 1.411 & 1.383 & 1.401 & -2.117 \\
\hline & 1.331 & 1.286 & 1.439 & 1.416 & 1.381 & 1.422 & 1.372 & 1.419 & -1.427 \\
\hline & 1.316 & 1.272 & 1.454 & 1.424 & 1.37 & 1.435 & 1.37 & 1.429 & -1.023 \\
\hline & 1.321 & 1.268 & 1.445 & 1.423 & 1.376 & 1.43 & 1.37 & 1.416 & -1.132 \\
\hline & 1.332 & 1.273 & 1.438 & 1.427 & 1.378 & 1.424 & 1.374 & 1.411 & -1.296 \\
\hline 1 and 2 avg & 1.345 & 1.32 & 1.423 & 1.401 & 1.392 & 1.4135 & 1.3835 & 1.401 & -2.063 \\
\hline 3 and 4 avg & 1.323 & 1.279 & 1.4465 & 1.42 & 1.3755 & 1.4285 & 1.371 & 1.424 & -1.225 \\
\hline 5 and 6 avg & 1.326 & 1.270 & 1.441 & 1.425 & 1.377 & 1.427 & 1.372 & 1.4135 & -1.214 \\
\hline overall avg & 1.331 & 1.289 & 1.437 & 1.415 & 1.381 & 1.423 & 1.3755 & 1.4128 & -1.501 \\
\hline
\end{tabular}

Table S7. Metrical Oxidation States for Ligands in $\mathrm{Np}\left(\mathrm{DOPO}^{\mathrm{q}}\right)_{2}\left(\mathrm{DOPO}^{\mathrm{sq}}\right)$.

\begin{tabular}{|r|r|r|r|r|r|r|r|r|r|}
\hline & C1-N & \multicolumn{1}{|c|}{ C2-O } & C1-C2 & C2-C3 & C3-C4 & C4-C5 & C5-C6 & C6-C1 & MOS Total \\
\hline & 1.373 & 1.324 & 1.426 & 1.471 & 1.396 & 1.447 & 1.358 & 1.363 & -1.837 \\
\hline & 1.368 & 1.348 & 1.402 & 1.406 & 1.389 & 1.418 & 1.380 & 1.407 & -2.421 \\
\hline & 1.324 & 1.278 & 1.451 & 1.437 & 1.384 & 1.423 & 1.360 & 1.416 & -1.158 \\
\hline & 1.322 & 1.266 & 1.462 & 1.426 & 1.390 & 1.445 & 1.347 & 1.451 & -0.904 \\
\hline & 1.336 & 1.264 & 1.430 & 1.447 & 1.381 & 1.423 & 1.364 & 1.428 & -0.984 \\
\hline & 1.329 & 1.285 & 1.449 & 1.440 & 1.379 & 1.428 & 1.389 & 1.398 & -1.433 \\
\hline 1 and 2 avg & 1.371 & 1.336 & 1.439 & 1.393 & 1.433 & 1.369 & 1.385 & 1.419 & -2.129 \\
\hline 3 and 4 avg & 1.323 & 1.272 & 1.432 & 1.387 & 1.434 & 1.354 & 1.434 & 1.457 & -1.031 \\
\hline 5 and 6 avg & 1.333 & 1.275 & 1.444 & 1.380 & 1.426 & 1.377 & 1.413 & 1.440 & -1.209 \\
\hline overall avg & 1.342 & 1.294 & 1.438 & 1.387 & 1.431 & 1.366 & 1.411 & 1.438 & -1.456 \\
\hline
\end{tabular}

Table S8. Metrical Oxidation States for Ligands in $\mathrm{U}\left(\mathrm{DOPO}^{\mathrm{q}}\right)_{2}\left(\mathrm{DOPO}^{\mathrm{sq}}\right)$.

\begin{tabular}{|l|c|c|c|c|c|c|c|c|c|}
\hline & C1-N & C2-O & C1-C2 & C2-C3 & C3-C4 & C4-C5 & C5-C6 & C1-C6 & MOS Total \\
\hline & 1.339 & 1.325 & 1.411 & 1.411 & 1.385 & 1.432 & 1.387 & 1.406 & -1.993 \\
\hline & 1.348 & 1.347 & 1.408 & 1.407 & 1.397 & 1.405 & 1.387 & 1.402 & -2.389 \\
\hline & 1.313 & 1.307 & 1.43 & 1.409 & 1.386 & 1.412 & 1.372 & 1.422 & -1.618 \\
\hline & 1.327 & 1.291 & 1.431 & 1.405 & 1.396 & 1.422 & 1.375 & 1.428 & -1.564 \\
\hline & 1.319 & 1.285 & 1.42 & 1.424 & 1.364 & 1.428 & 1.37 & 1.407 & -1.357 \\
\hline & 1.358 & 1.313 & 1.438 & 1.407 & 1.386 & 1.408 & 1.387 & 1.384 & -2.033 \\
\hline 1 and 2 avg & 1.3435 & 1.336 & 1.4095 & 1.409 & 1.391 & 1.4185 & 1.387 & 1.404 & -2.191 \\
\hline 3 and 4 avg & 1.32 & 1.299 & 1.4305 & 1.407 & 1.391 & 1.417 & 1.3735 & 1.425 & -1.591 \\
\hline 5 and 6 avg & 1.3385 & 1.299 & 1.429 & 1.4155 & 1.375 & 1.418 & 1.3785 & 1.3955 & -1.695 \\
\hline overall avg & 1.334 & 1.311 & 1.423 & 1.410 & 1.385 & 1.417 & 1.379 & 1.408 & -1.826 \\
\hline
\end{tabular}


Table S9. Metrical Oxidation States for Ligands in $\mathrm{Th}\left(\mathrm{DOPO}^{\mathrm{q}}\right)_{2}\left(\mathrm{DOPO}^{\mathrm{sq}}\right)$.

\begin{tabular}{|l|c|c|c|c|c|c|c|c|c|}
\hline & C1-N & C2-O & C1-C2 & C2-C3 & C3-C4 & C4-C5 & C5-C6 & C1-C6 & $\begin{array}{c}\text { MOS } \\
\text { Total }\end{array}$ \\
\hline & 1.344 & 1.289 & 1.422 & 1.427 & 1.388 & 1.405 & 1.396 & 1.422 & -1.698 \\
\hline & 1.321 & 1.282 & 1.453 & 1.416 & 1.389 & 1.431 & 1.35 & 1.424 & -1.189 \\
\hline & 1.352 & 1.316 & 1.43 & 1.415 & 1.396 & 1.419 & 1.381 & 1.401 & -1.958 \\
\hline & 1.357 & 1.323 & 1.426 & 1.402 & 1.4 & 1.399 & 1.402 & 1.396 & -2.278 \\
\hline & 1.333 & 1.273 & 1.444 & 1.421 & 1.379 & 1.434 & 1.375 & 1.432 & -1.215 \\
\hline & 1.323 & 1.286 & 1.447 & 1.428 & 1.377 & 1.425 & 1.376 & 1.412 & -1.309 \\
\hline 1 and 2 avg & 1.3325 & 1.2855 & 1.4375 & 1.4215 & 1.3885 & 1.418 & 1.373 & 1.423 & -1.444 \\
\hline 3 and 4 avg & 1.3545 & 1.3195 & 1.428 & 1.4085 & 1.398 & 1.409 & 1.3915 & 1.3985 & -2.118 \\
\hline 5 and 6 avg & 1.328 & 1.2795 & 1.4455 & 1.4245 & 1.378 & 1.4295 & 1.3755 & 1.422 & -1.262 \\
\hline overall avg & 1.338 & 1.294 & 1.437 & 1.418 & 1.388 & 1.418 & 1.38 & 1.414 & -1.608 \\
\hline
\end{tabular}

Table S10. Metrical Oxidation States for Ligands in $\mathrm{Hf}\left(\mathrm{DOPO}^{\mathrm{q}}\right)_{2}\left(\mathrm{DOPO}^{\mathrm{sq}}\right)$.

\begin{tabular}{|l|c|c|c|c|c|c|c|c|c|}
\hline & C1-N & C2-O & C1-C2 & C2-C3 & C3-C4 & C4-C5 & C5-C6 & C1-C6 & $\begin{array}{c}\text { MOS } \\
\text { Total }\end{array}$ \\
\hline & 1.337 & 1.273 & 1.439 & 1.423 & 1.39 & 1.442 & 1.369 & 1.422 & -1.265 \\
\hline & 1.303 & 1.269 & 1.436 & 1.422 & 1.375 & 1.446 & 1.37 & 1.431 & -0.995 \\
\hline & 1.343 & 1.317 & 1.417 & 1.416 & 1.389 & 1.418 & 1.398 & 1.395 & -2.028 \\
\hline & 1.34 & 1.308 & 1.41 & 1.418 & 1.389 & 1.429 & 1.39 & 1.403 & -1.876 \\
\hline & 1.336 & 1.271 & 1.439 & 1.43 & 1.368 & 1.434 & 1.371 & 1.41 & -1.208 \\
\hline & 1.308 & 1.278 & 1.433 & 1.43 & 1.369 & 1.438 & 1.379 & 1.429 & -1.115 \\
\hline 1 and 2 avg & 1.32 & 1.271 & 1.4375 & 1.4225 & 1.3825 & 1.444 & 1.3695 & 1.4265 & -1.130 \\
\hline 3 and 4 avg & 1.3415 & 1.3125 & 1.4135 & 1.417 & 1.389 & 1.4235 & 1.394 & 1.399 & -1.952 \\
\hline 5 and 6 avg & 1.322 & 1.2745 & 1.436 & 1.43 & 1.3685 & 1.436 & 1.375 & 1.4195 & -1.162 \\
\hline overall avg & 1.327 & 1.286 & 1.429 & 1.423 & 1.38 & 1.434 & 1.379 & 1.415 & -1.414 \\
\hline
\end{tabular}




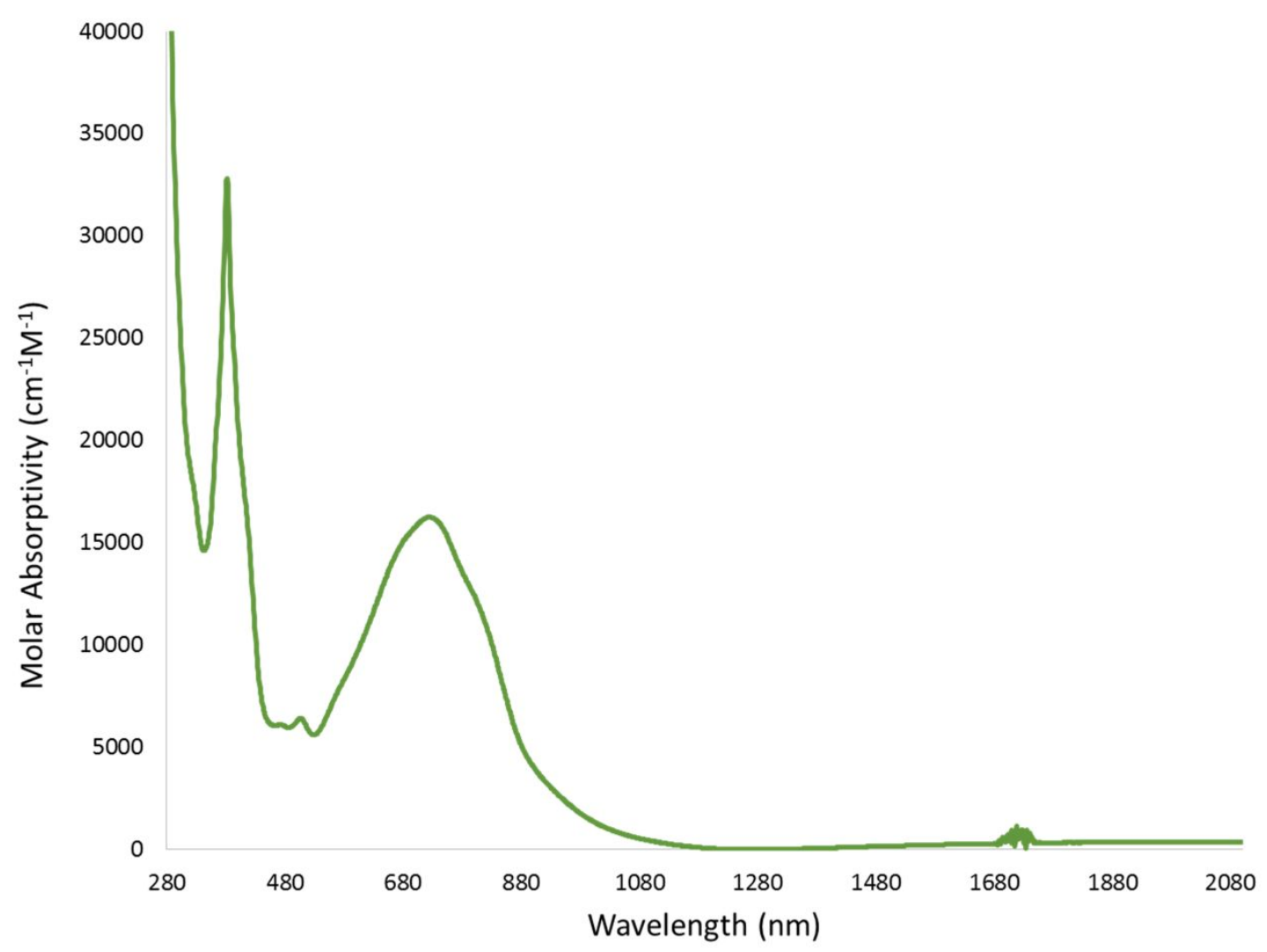

Figure S1. Solution UV-Vis-NIR spectrum of $\left.\mathrm{U}_{(\mathrm{DOPO}}\right)_{2}\left(\mathrm{DOPO}^{\mathrm{sq}}\right)$ in THF at $25^{\circ} \mathrm{C}$. Solvent overtones are present at 280 and $1700 \mathrm{~nm}$. 


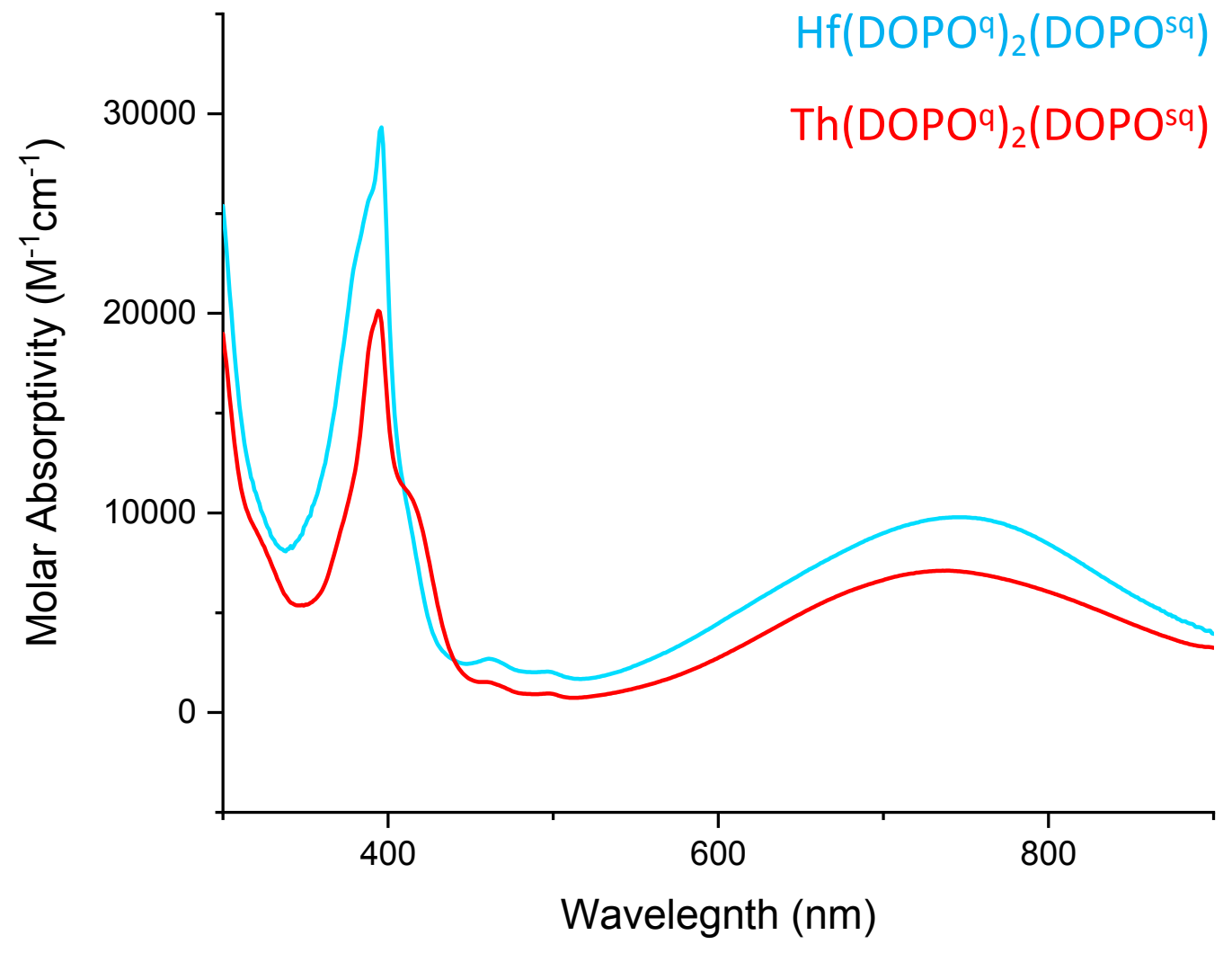

Figure S2. Solution UV-Vis-NIR spectrum of $\mathrm{M}\left(\mathrm{DOPO}^{\mathrm{q}}\right)_{2}\left(\mathrm{DOPO}^{\mathrm{sq}}\right)(\mathrm{M}$ : Hf, Th) in THF at $25^{\circ} \mathrm{C}$. Solvent overtones are present at $280 \mathrm{~nm}$. 


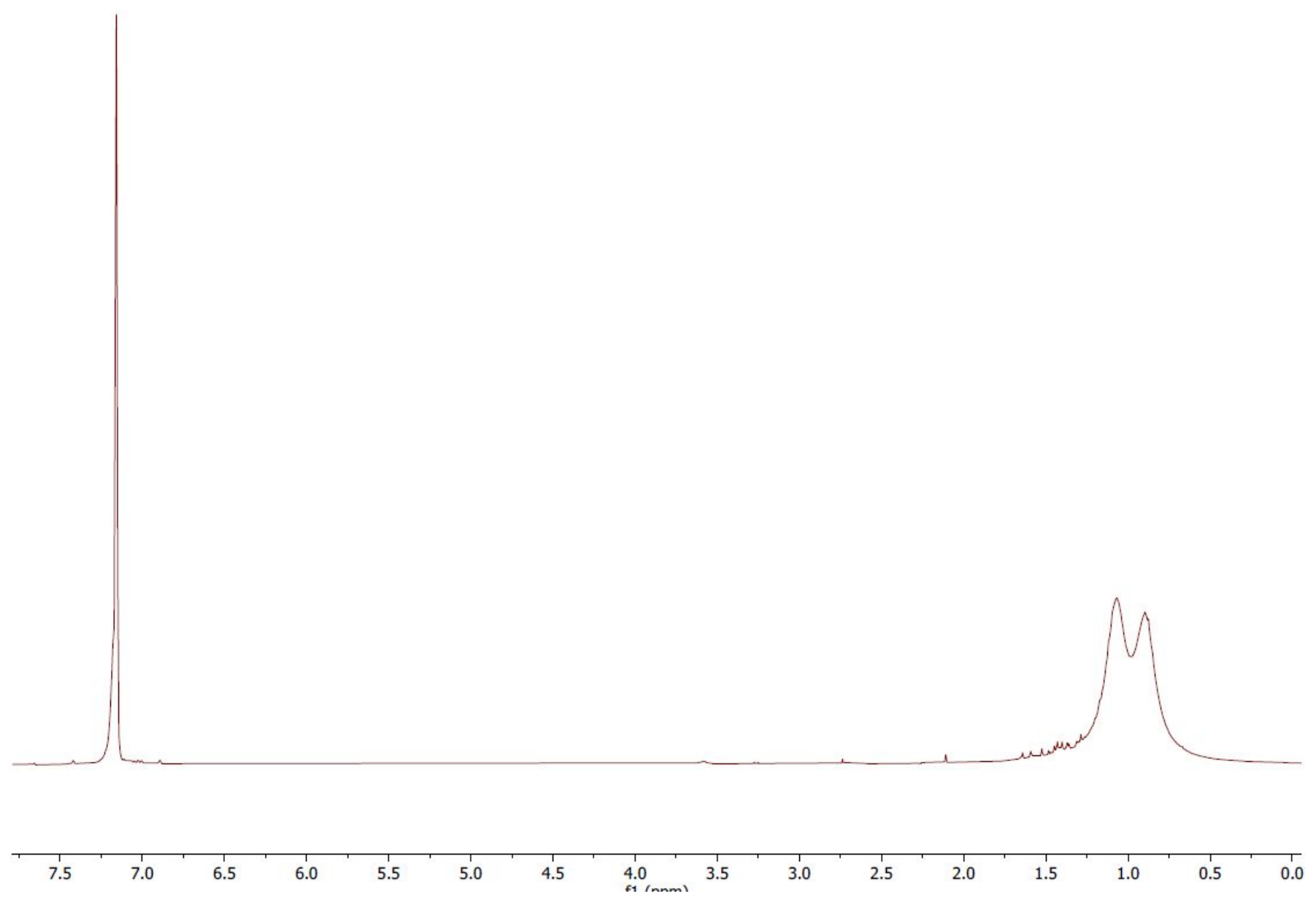

Figure S3. ${ }^{1} \mathrm{H}$ NMR spectrum of $\mathrm{Hf}\left(\mathrm{DOPO}^{\mathrm{q}}\right)_{2}\left(\mathrm{DOPO}^{\mathrm{sq}}\right)$ in $\mathrm{C}_{6} \mathrm{D}_{6}$ at $25^{\circ} \mathrm{C}$. 

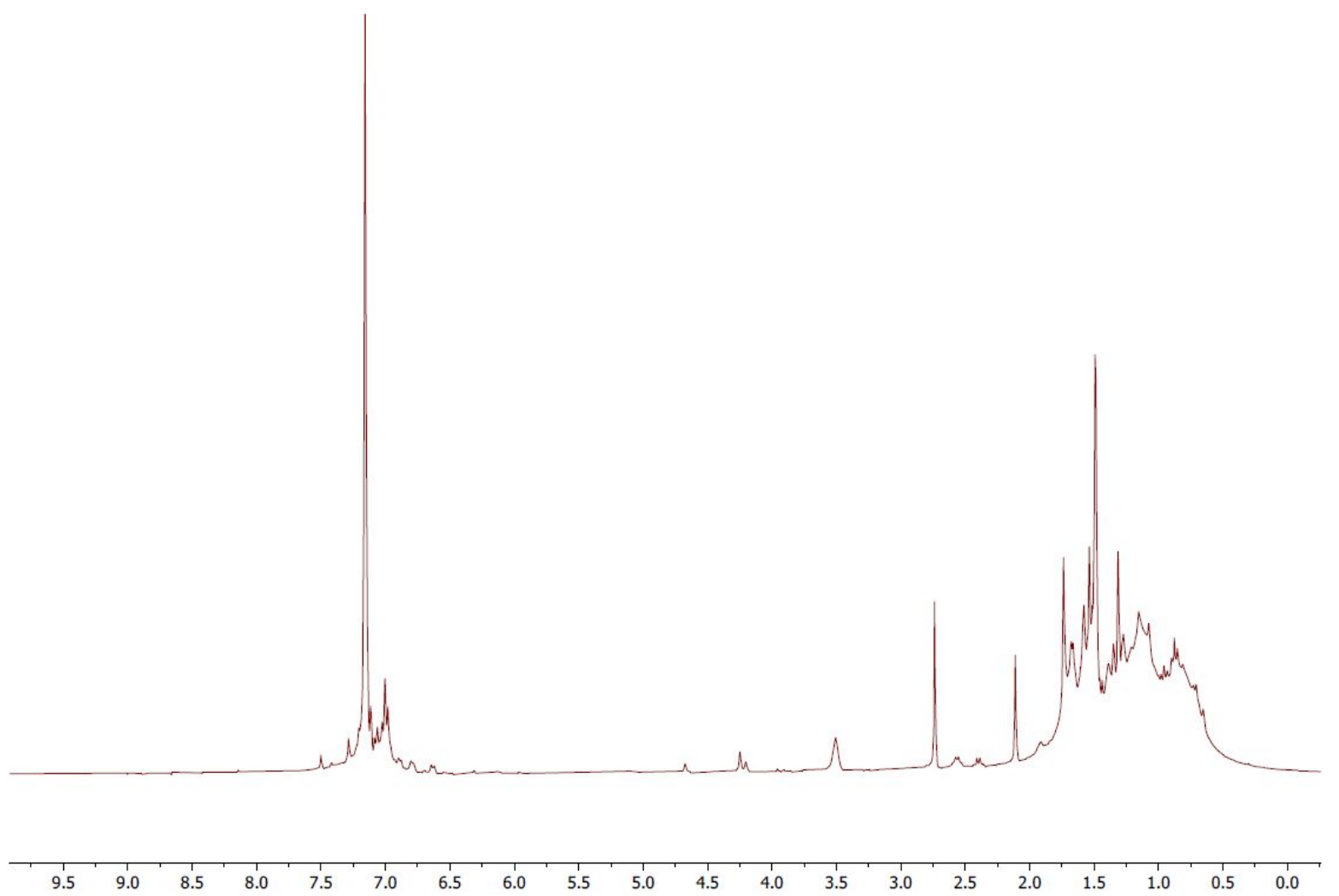

Figure S4. ${ }^{1} \mathrm{H}$ NMR spectrum of $\mathrm{Th}\left(\mathrm{DOPO}^{\mathrm{q}}\right)_{2}\left(\mathrm{DOPO}^{\mathrm{sq}}\right)$ in $\mathrm{C}_{6} \mathrm{D}_{6}$ at $25^{\circ} \mathrm{C}$. 


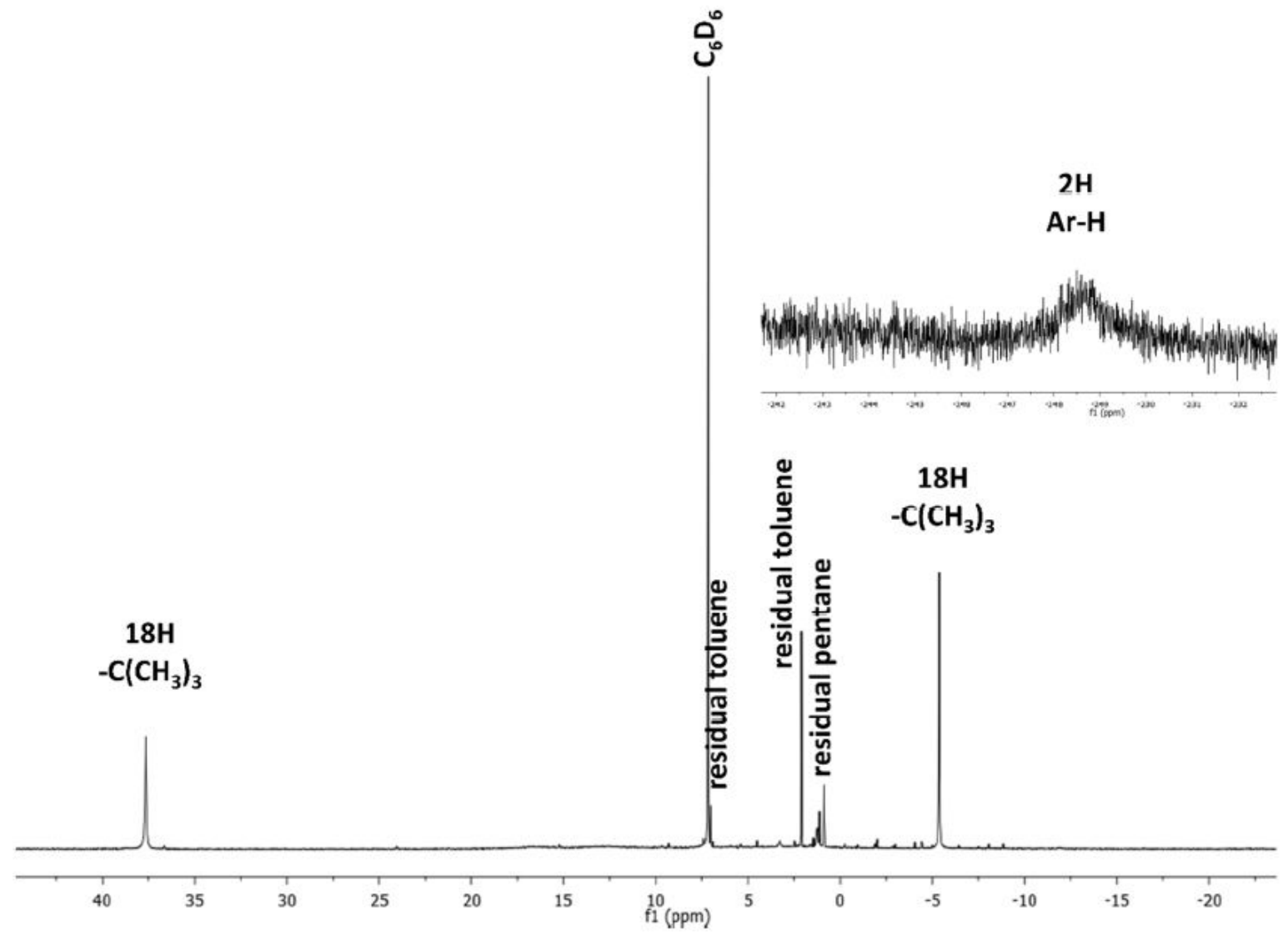

Figure S5. ${ }^{1} \mathrm{H}$ NMR spectrum of $\mathrm{U}\left(\mathrm{DOPO}^{\mathrm{q}}\right)_{2}\left(\mathrm{DOPO}^{\mathrm{sq}}\right)$ in $\mathrm{C}_{6} \mathrm{D}_{6}$ at $25^{\circ} \mathrm{C}$. 


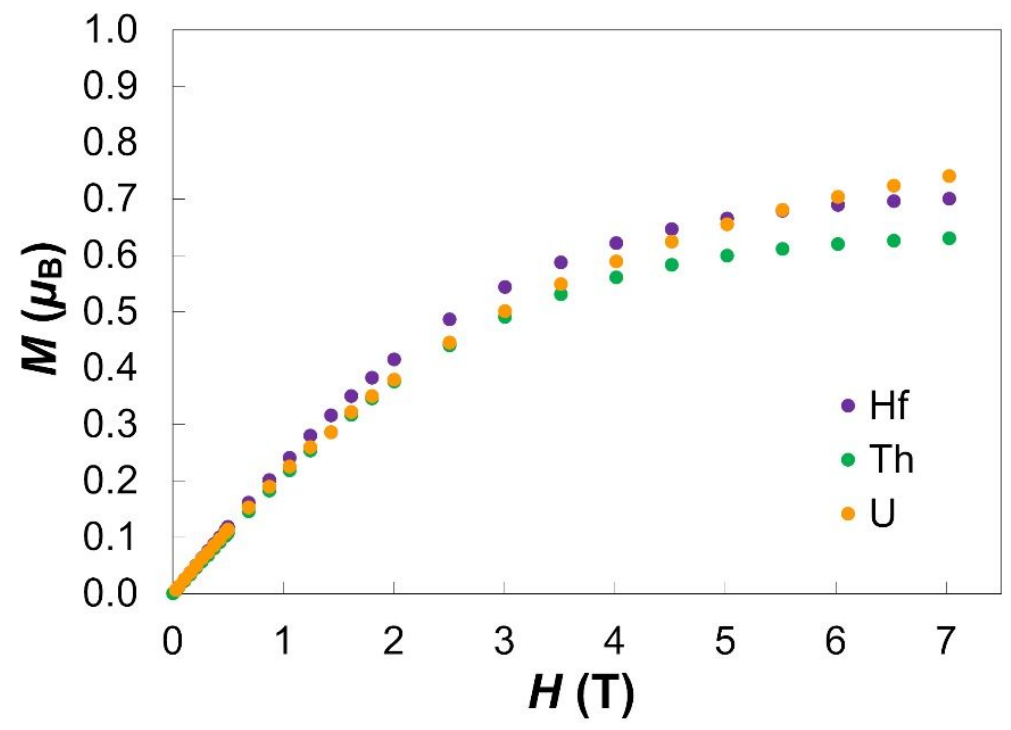

Figure S6. Field-dependent magnetic data collected at $2 \mathrm{~K}$ for $\mathrm{Hf}\left(\mathrm{DOPO}^{\mathrm{q}}\right)_{2}\left(\mathrm{DOPO}^{\mathrm{sq}}\right)$ (purple), $\mathrm{Th}\left(\mathrm{DOPO}^{\mathrm{q}}\right)_{2}\left(\mathrm{DOPO}^{\mathrm{sq}}\right)$ (green), and $\mathrm{U}\left(\mathrm{DOPO}^{\mathrm{q}}\right)_{2}\left(\mathrm{DOPO}^{\mathrm{sq}}\right)$ (orange). 
Table S11: Absolute energy (Hartree) and relative energy ( $\mathrm{kcal} / \mathrm{mol})$ of various spin states of M-DOPO complexes using CASSCF and CASPT2 level of theory.

CASSCF

CASPT2

\begin{tabular}{|c|c|c|c|c|c|}
\hline $\begin{array}{c}\text { Metal } \\
\text { complex }\end{array}$ & Spin State & $\begin{array}{c}\text { Absolute } \\
\text { Energy } \\
\text { (Hartree) }\end{array}$ & $\begin{array}{c}\text { Relative } \\
\text { Energy } \\
\text { (kcal/mol) }\end{array}$ & $\begin{array}{c}\text { Absolute } \\
\text { Energy } \\
\text { (Hartree) }\end{array}$ & $\begin{array}{c}\text { Relative } \\
\text { Energy } \\
\text { (kcal/mol) }\end{array}$ \\
\hline \multirow{2}{*}{ U-DOPO } & Quartet & -30161.833943 & 0.1 & -30167.944529 & 0.5 \\
\cline { 2 - 7 } & Doublet & -30161.834088 & 0.0 & -30167.945379 & 0.0 \\
\hline \multirow{2}{*}{ Np-DOPO } & Quintet & -30948.875992 & 0.0 & -30955.019763 & 0.3 \\
\cline { 2 - 7 } & Triplet & -30948.875592 & 0.2 & -30955.020223 & 0.0 \\
\cline { 2 - 7 } & Singlet & -30948.822869 & 33.3 & -30954.983107 & 23.3 \\
\hline \multirow{2}{*}{ Pu-DOPO } & Sextet & -31751.271428 & 0.0 & -31757.479487 & 0.0 \\
\cline { 2 - 7 } & Quartet & -31751.271011 & 0.3 & -31757.479376 & 0.1 \\
\hline & Doublet & -31751.201199 & 44.1 & -31757.419567 & 37.6 \\
\hline \multirow{2}{*}{ Hf-DOPO } & Doublet & -17276.157086 & 0.0 & - & - \\
\hline \multirow{2}{*}{ Th-DOPO } & Doublet & -28632.758871 & 0.0 & - & - \\
\hline
\end{tabular}

Table S12: LoProp charge and Mulliken spin density on the metal and radical $\mathrm{N}$ center obtained from CASSCF level of theory. N.B.: The concept of spin density is not valid for singlet state.

LoProp Charge

Mulliken Spin Density

\begin{tabular}{|c|c|c|c|c|c|}
\hline $\begin{array}{c}\text { Metal } \\
\text { complex }\end{array}$ & Spin State & Metal Center & $\begin{array}{c}\text { Radical N } \\
\text { Center }\end{array}$ & Metal Center & $\begin{array}{c}\text { Radical N } \\
\text { Center }\end{array}$ \\
\hline \multirow{2}{*}{ U-DOPO } & Quartet & 3.1376 & -0.3504 & 1.9916 & 0.5510 \\
\cline { 2 - 6 } & Doublet & 3.1383 & -0.3497 & 1.3095 & -0.1817 \\
\hline \multirow{2}{*}{ Np-DOPO } & Quintet & 3.1770 & -0.3645 & 2.9952 & 0.5873 \\
\cline { 2 - 6 } & Triplet & 3.1786 & -0.3628 & 2.4666 & -0.2949 \\
\cline { 2 - 6 } & Singlet & 3.1738 & -0.3597 & - & - \\
\hline \multirow{2}{*}{ Pu-DOPO } & Sextet & 3.1895 & -0.3785 & 3.9880 & 0.5189 \\
\cline { 2 - 6 } & Quartet & 3.1914 & -0.3760 & 3.5556 & -0.3130 \\
\cline { 2 - 6 } & Doublet & 3.1880 & -0.3770 & 1.3118 & -0.1733 \\
\hline \multirow{2}{*}{ Hf-DOPO } & Doublet & 1.8640 & -0.2038 & 0.0151 & 0.5461 \\
\hline Th-DOPO & Doublet & 2.5981 & -0.3171 & 0.0214 & 0.5371 \\
\hline
\end{tabular}


Table S13: Dominant configurations (with weight > 0.01) of each spin state of M-DOPO complexes using CASSCF level of theory. Doubly occupied orbitals have occupation number of 2 , singly occupied orbitals have occupation number 1 (1 $\mathrm{u}$ and $1 \mathrm{~d}$ refers to up and down spin electrons respectively) and unoccupied orbitals have occupation number 0 .

\begin{tabular}{|c|c|c|c|}
\hline $\begin{array}{l}\text { Metal } \\
\text { complex }\end{array}$ & Spin State & Electronic Configuration & Weight \\
\hline \multirow[t]{10}{*}{ U-DOPO } & \multirow[t]{4}{*}{ Quartet } & $\pi_{1}^{2} \pi_{2}^{2} \pi_{3}^{1} u \pi_{4}^{0} f_{1}^{1 u} f_{2}^{1 u} f_{3}^{0} f_{4}^{0} f_{5}^{0} \pi_{5}^{0} f_{6}^{0} f_{7}^{0}$ & 0.873 \\
\hline & & $\pi_{1}^{2} \pi_{2}^{0} \pi_{3}^{1 u} \pi_{4}^{2} f_{1}^{1 u} f_{2}^{1 u} f_{3}^{0} f_{4}^{0} f_{5}^{0} \pi_{5}^{0} f_{6}^{0} f_{7}^{0}$ & 0.019 \\
\hline & & $\pi_{1}^{2} \pi_{2}^{2} \pi_{3}^{1} u \pi_{4}^{0} f_{1}^{0} f_{2}^{0} f_{3}^{0} f_{4}^{0} f_{5}^{1} u \pi_{5}^{0} f_{6}^{0} f_{7}^{1 u}$ & 0.050 \\
\hline & & $\pi_{1}^{0} \pi_{2}^{2} \pi_{3}^{1} u \pi_{4}^{0} f_{1}^{1 u} f_{2}^{1} u f_{3}^{0} f_{4}^{0} f_{5}^{0} \pi_{5}^{2} f_{6}^{0} f_{7}^{0}$ & 0.032 \\
\hline & Doublet & $\pi_{1}^{2} \pi_{2}^{2} \pi_{3}^{2} \pi_{4}^{0} f_{1}^{0} f_{2}^{1 u} f_{3}^{0} f_{4}^{0} f_{5}^{0} \pi_{5}^{0} f_{6}^{0} f_{7}^{0}$ & 0.327 \\
\hline & & $\pi_{1}^{2} \pi_{2}^{2} \pi_{3}^{1 u} \pi_{4}^{0} f_{1}^{1 d} f_{2}^{1 u} f_{3}^{0} f_{4}^{0} f_{5}^{0} \pi_{5}^{0} f_{6}^{0} f_{7}^{0}$ & 0.084 \\
\hline & & $\pi_{1}^{2} \pi_{2}^{2} \pi_{3}^{0} \pi_{4}^{0} f_{1}^{2} f_{2}^{1 u} f_{3}^{0} f_{4}^{0} f_{5}^{0} \pi_{5}^{0} f_{6}^{0} f_{7}^{0}$ & 0.244 \\
\hline & & $\pi_{1}^{2} \pi_{2}^{2} \pi_{3}^{1} u \pi_{4}^{0} f_{1}^{1 u} f_{2}^{1 d} f_{3}^{0} f_{4}^{0} f_{5}^{0} \pi_{5}^{0} f_{6}^{0} f_{7}^{0}$ & 0.204 \\
\hline & & $\pi_{1}^{2} \pi_{2}^{2} \pi_{3}^{1} u \pi_{4}^{0} f_{1}^{0} f_{2}^{0} f_{3}^{0} f_{4}^{0} f_{5}^{1} d^{1} \pi_{5}^{1 u} f_{6}^{0} f_{7}^{0}$ & 0.019 \\
\hline & & $\pi_{1}^{2} \pi_{2}^{2} \pi_{3}^{1} u \pi_{4}^{0} f_{1}^{0} f_{2}^{0} f_{3}^{0} f_{4}^{0} f_{5}^{1} \pi_{5}^{0} f_{6}^{0} f_{7}^{1 u}$ & 0.018 \\
\hline \multirow[t]{13}{*}{ Np-DOPO } & \multirow[t]{4}{*}{ Quintet } & $\pi_{1}^{2} \pi_{2}^{2} f_{1}^{0} f_{2}^{1 u} f_{3}^{0} f_{4}^{1 u} f_{5}^{0} f_{6}^{0} f_{7}^{1 u} \pi_{3}^{1 u} \pi_{4}^{0} \pi_{5}^{0}$ & 0.859 \\
\hline & & $\pi_{1}^{2} \pi_{2}^{2} f_{1}^{1 u} f_{2}^{0} f_{3}^{0} f_{4}^{0} f_{5}^{1 u} f_{6}^{0} f_{7}^{1} u \pi_{3}^{1 u} \pi_{4}^{0} \pi_{5}^{0}$ & 0.015 \\
\hline & & $\pi_{1}^{2} \pi_{2}^{0} f_{1}^{0} f_{2}^{1} u f_{3}^{0} f_{4}^{1 u} f_{5}^{0} f_{6}^{0} f_{7}^{1} u \pi_{3}^{1 u} \pi_{4}^{0} \pi_{5}^{2}$ & 0.027 \\
\hline & & $\pi_{1}^{0} \pi_{2}^{2} f_{1}^{0} f_{2}^{1} u f_{3}^{0} f_{4}^{1 u} f_{5}^{0} f_{6}^{0} f_{7}^{1} u \pi_{3}^{1 u} \pi_{4}^{2} \pi_{5}^{0}$ & 0.029 \\
\hline & \multirow[t]{4}{*}{ Triplet } & $\pi_{1}^{2} \pi_{2}^{2} f_{1}^{0} f_{2}^{1 u} f_{3}^{0} f_{4}^{1 u} f_{5}^{0} f_{6}^{0} f_{7}^{2} \pi_{3}^{0} \pi_{4}^{0} \pi_{5}^{0}$ & 0.224 \\
\hline & & $\pi_{1}^{2} \pi_{2}^{2} f_{1}^{0} f_{2}^{1 u} f_{3}^{0} f_{4}^{1 u} f_{5}^{0} f_{6}^{0} f_{7}^{1 d} \pi_{3}^{1 u} \pi_{4}^{0} \pi_{5}^{0}$ & 0.078 \\
\hline & & $\pi_{1}^{2} \pi_{2}^{2} f_{1}^{0} f_{2}^{1 u} f_{3}^{0} f_{4}^{1 u} f_{5}^{0} f_{6}^{0} f_{7}^{1} u \pi_{3}^{1 d} \pi_{4}^{0} \pi_{5}^{0}$ & 0.259 \\
\hline & & $\pi_{1}^{2} \pi_{2}^{2} f_{1}^{0} f_{2}^{1 u} f_{3}^{0} f_{4}^{1 u} f_{5}^{0} f_{6}^{0} f_{7}^{0} \pi_{3}^{2} \pi_{4}^{0} \pi_{5}^{0}$ & 0.281 \\
\hline & \multirow[t]{5}{*}{ Singlet } & $\pi_{1}^{2} \pi_{2}^{2} f_{1}^{2} f_{2}^{0} f_{3}^{1 u} f_{4}^{0} f_{5}^{0} f_{6}^{0} f_{7}^{0} \pi_{3}^{1 d} \pi_{4}^{0} \pi_{5}^{0}$ & 0.011 \\
\hline & & $\pi_{1}^{2} \pi_{2}^{2} f_{1}^{1 u} f_{2}^{1 d} f_{3}^{0} f_{4}^{0} f_{5}^{0} f_{6}^{1 u} f_{7}^{0} \pi_{3}^{1 d} \pi_{4}^{0} \pi_{5}^{0}$ & 0.012 \\
\hline & & $\pi_{1}^{2} \pi_{2}^{2} f_{1}^{0} f_{2}^{2} f_{3}^{0} f_{4}^{0} f_{5}^{0} f_{6}^{0} f_{7}^{2} \pi_{3}^{0} \pi_{4}^{0} \pi_{5}^{0}$ & 0.083 \\
\hline & & $\pi_{1}^{2} \pi_{2}^{2} f_{1}^{0} f_{2}^{2} f_{3}^{0} f_{4}^{0} f_{5}^{0} f_{6}^{0} f_{7}^{1} u \pi_{3}^{1 d} \pi_{4}^{0} \pi_{5}^{0}$ & 0.058 \\
\hline & & $\pi_{1}^{2} \pi_{2}^{2} f_{1}^{0} f_{2}^{2} f_{3}^{0} f_{4}^{0} f_{5}^{0} f_{6}^{0} f_{7}^{0} \pi_{3}^{2} \pi_{4}^{0} \pi_{5}^{0}$ & 0.118 \\
\hline
\end{tabular}




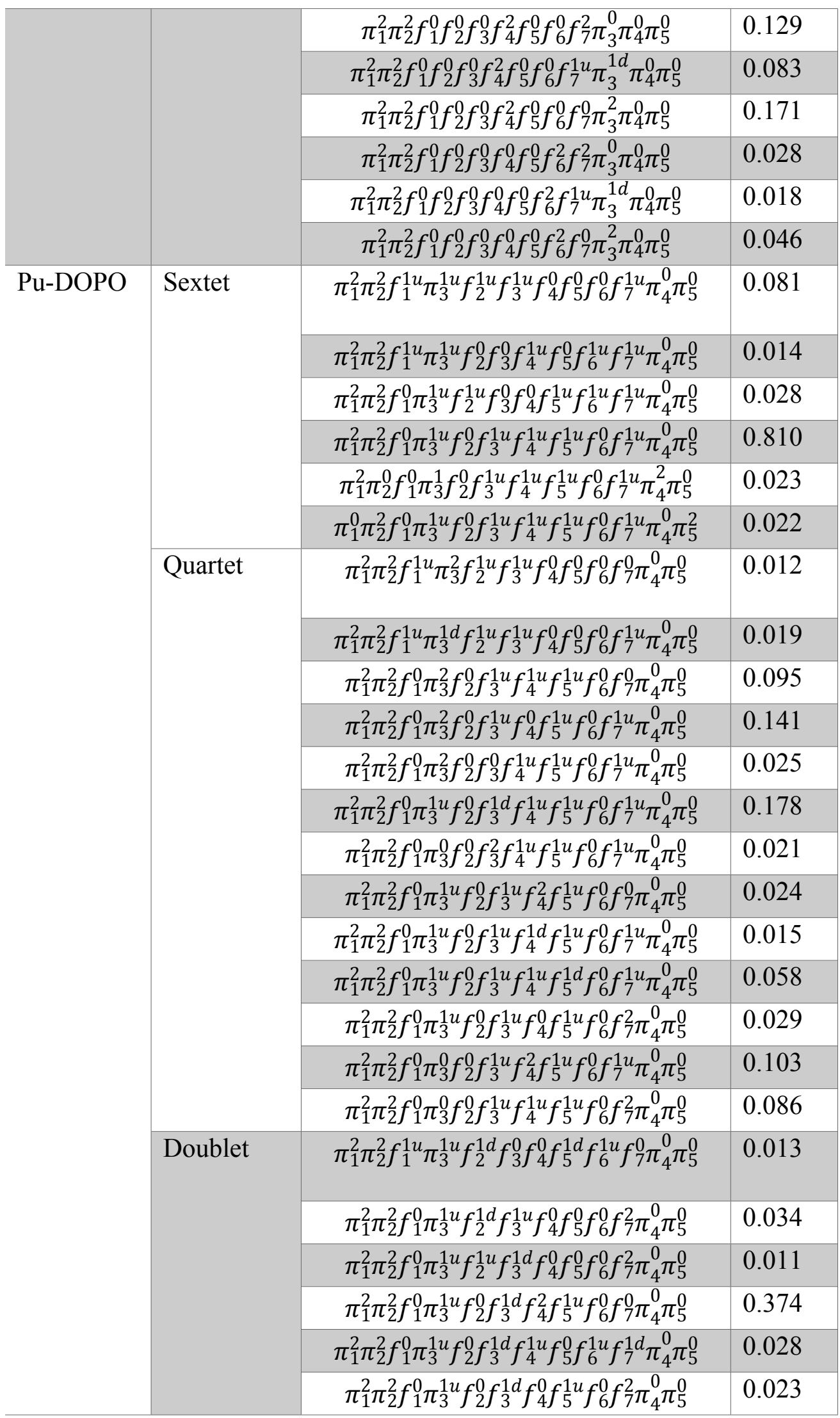




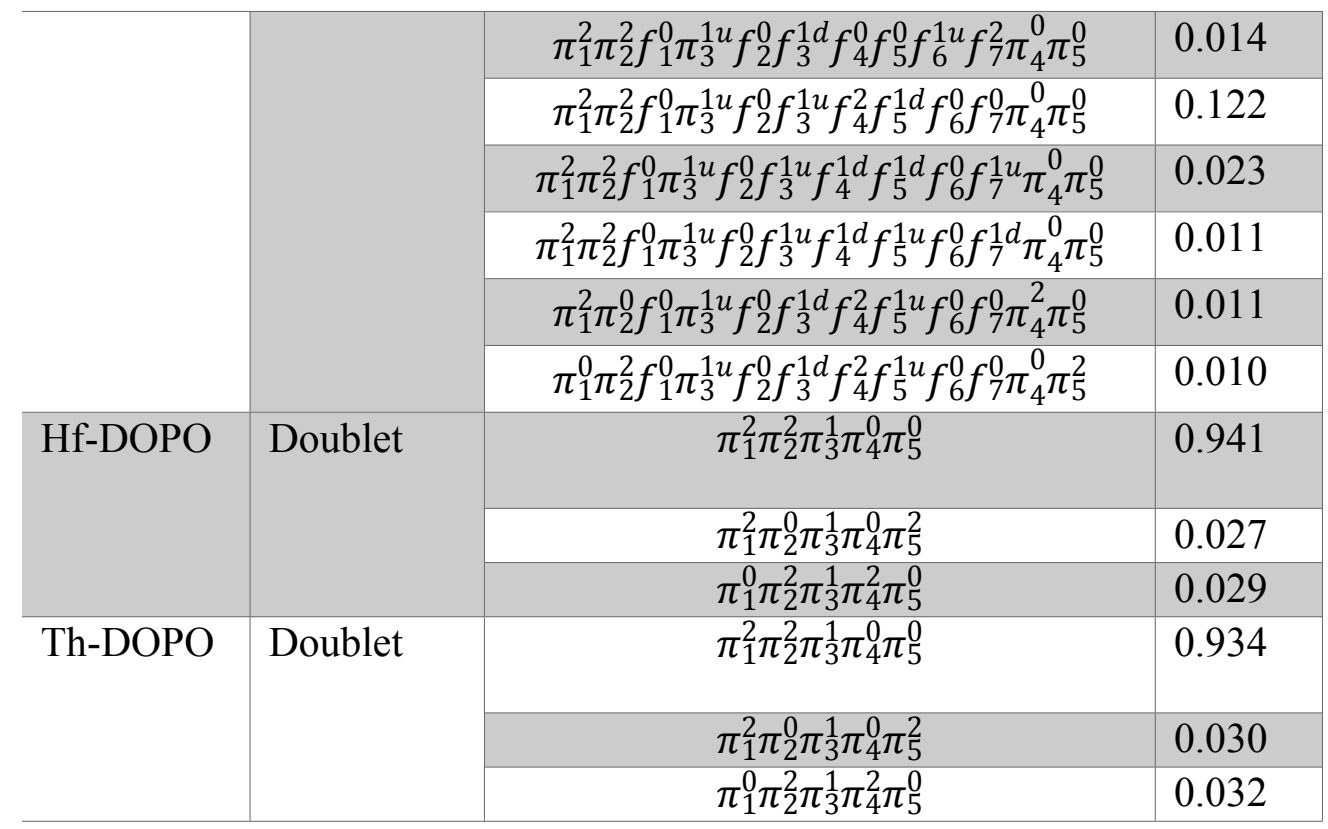




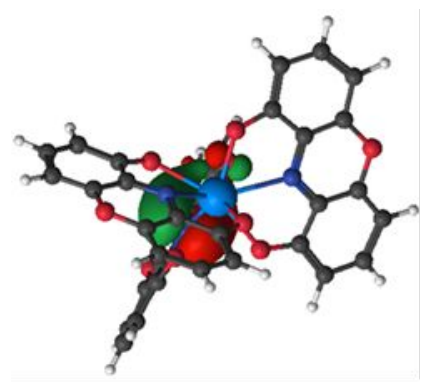

(1.92)

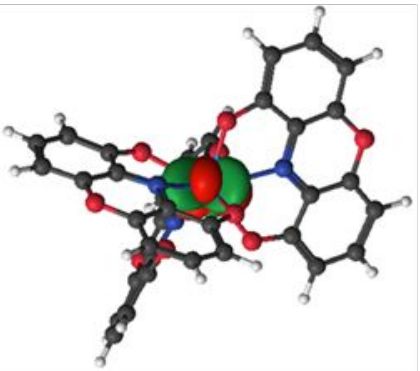

(0.94)

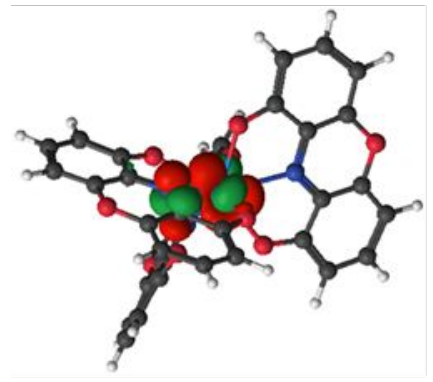

(0.06)

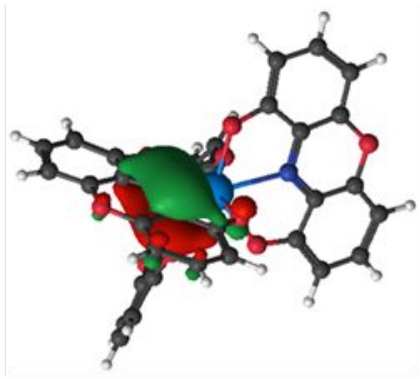

(1.94)

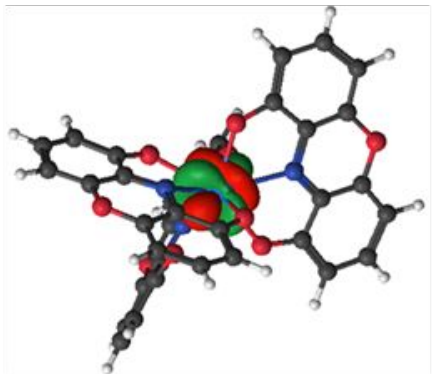

(0.94)

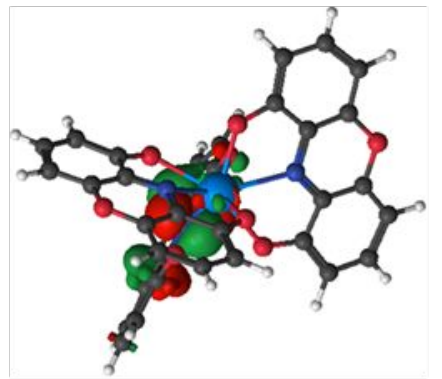

$(0.07)$

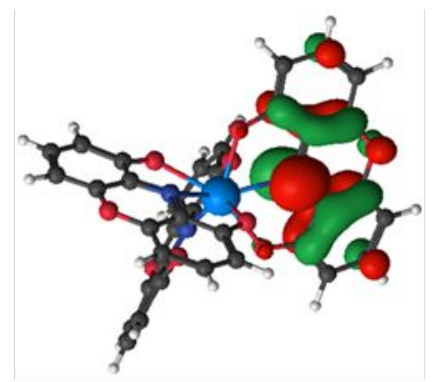

(1.00)

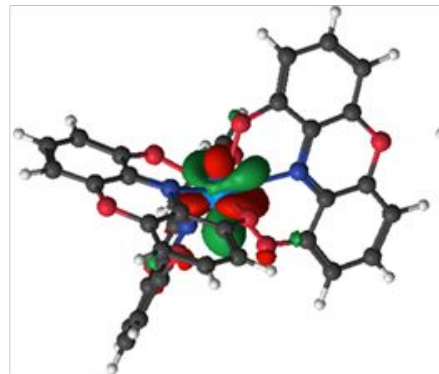

$(0.00)$

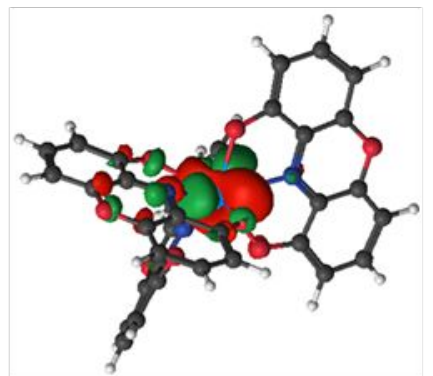

$(0.00)$

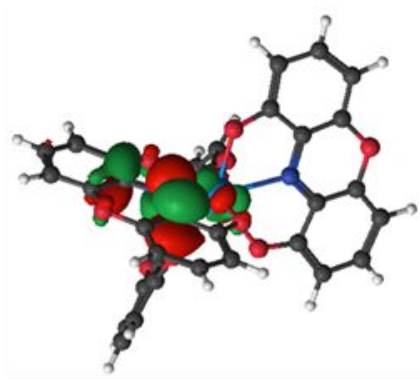

(0.06)

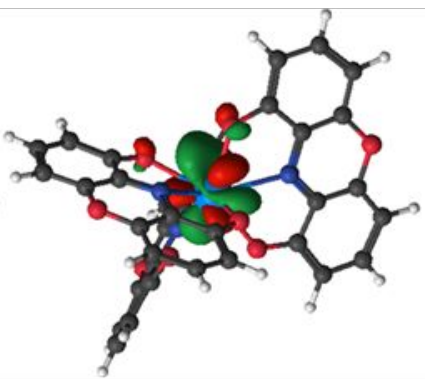

$(0.00)$

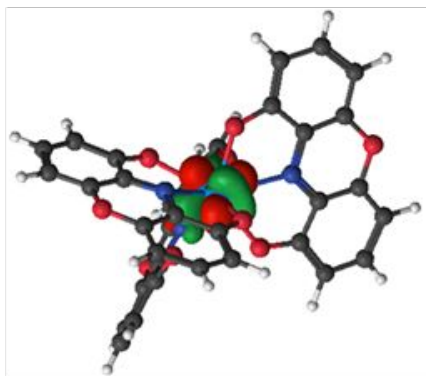

(0.06)

Figure S7: Active space orbitals of quartet spin state of $\mathrm{U}\left(\mathrm{DOPO}^{\mathrm{q}}\right)_{2}\left(\mathrm{DOPO}^{\mathrm{sq}}\right)$ (occupation numbers are in brackets). 


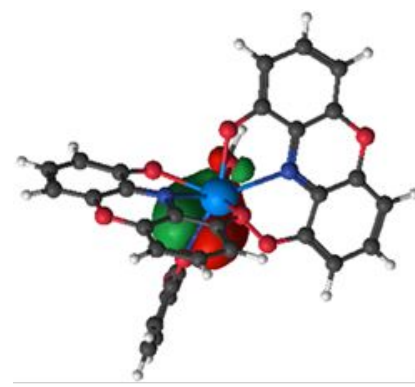

$(1.92)$

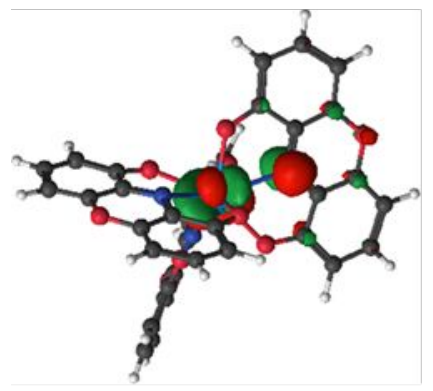

(0.86)

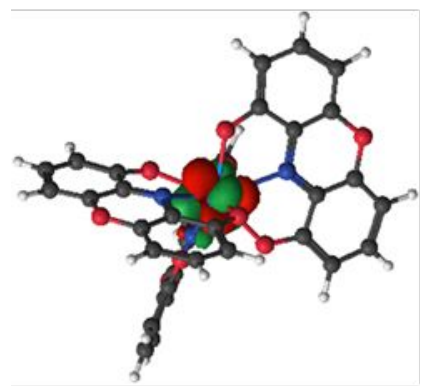

(0.07)

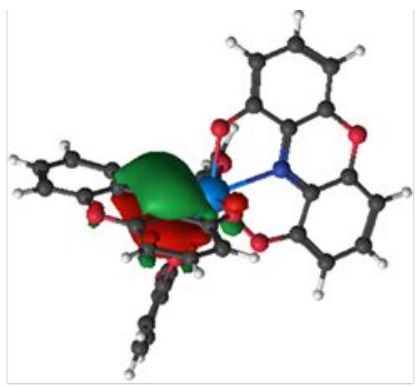

(1.94)

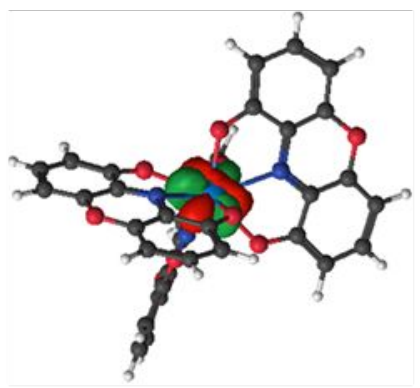

(0.93)

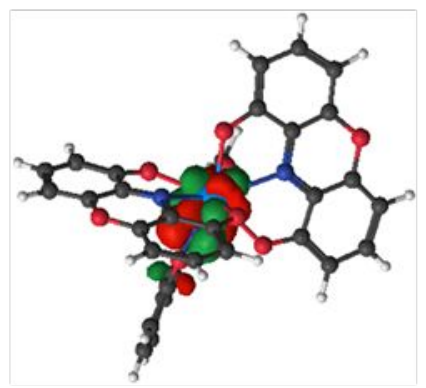

(0.08)

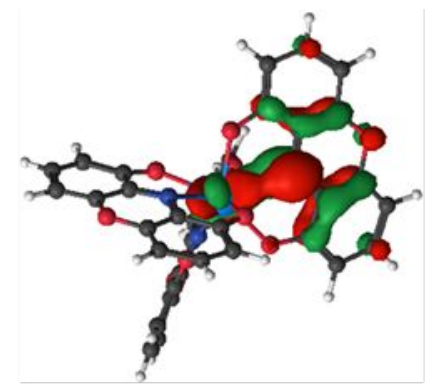

(1.07)

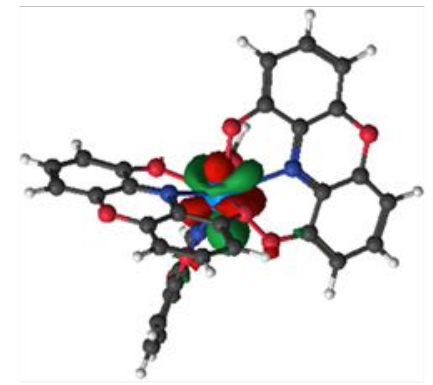

$(0.00)$
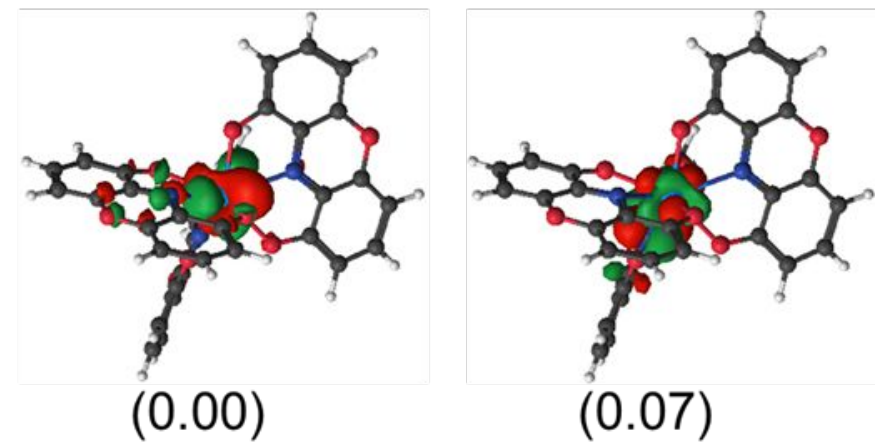

(0.07)

Figure S8: Active space orbitals of doublet spin state of $\mathrm{U}\left(\mathrm{DOPO}^{\mathrm{q}}\right)_{2}\left(\mathrm{DOPO}^{\mathrm{sq}}\right)$ (occupation numbers are in brackets). 


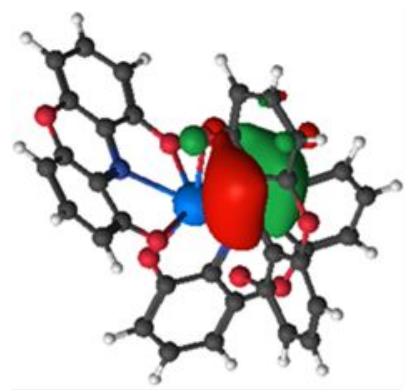

(1.93)

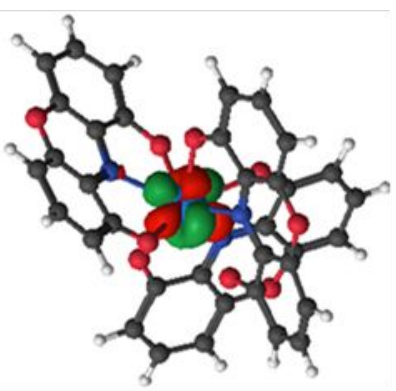

(0.04)

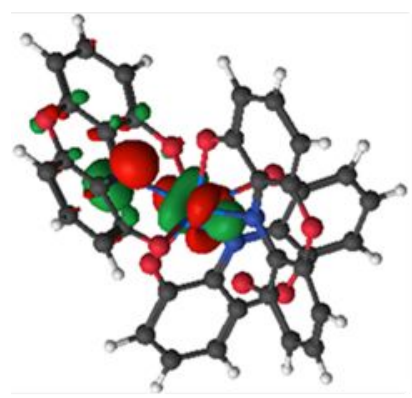

(0.90)

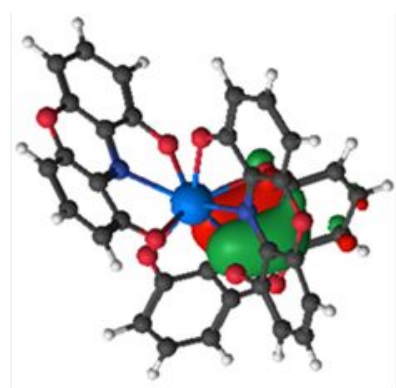

$(1.94)$

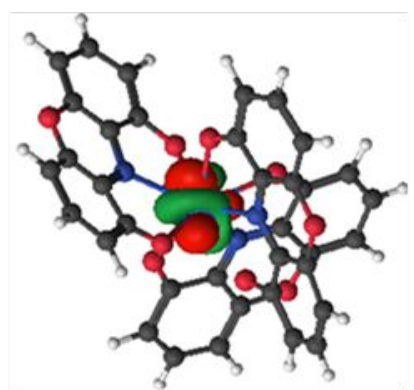

$(0.94)$

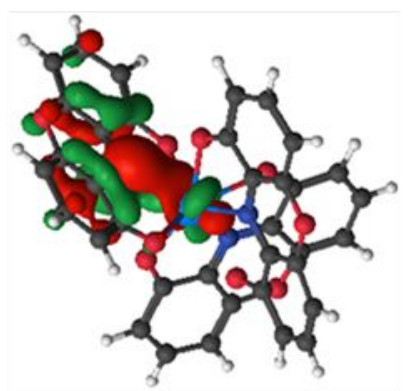

(1.05)

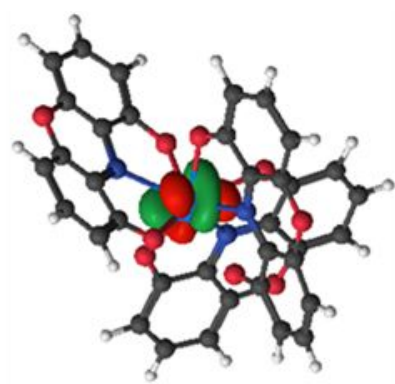

$(0.05)$

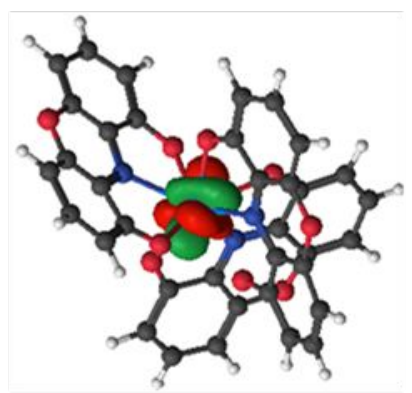

(0.05)

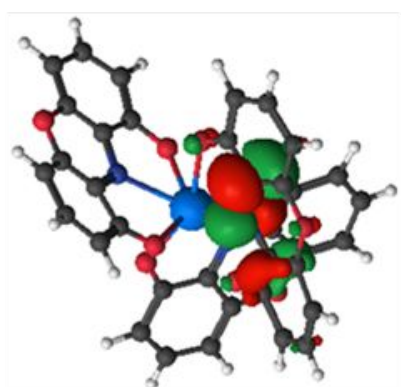

$(0.07)$

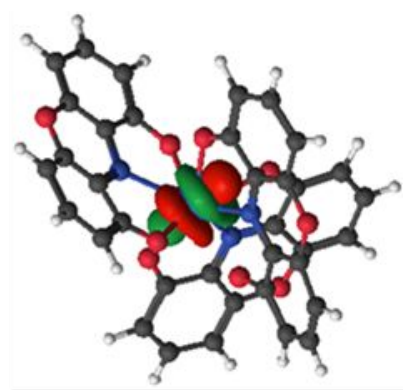

(0.94)

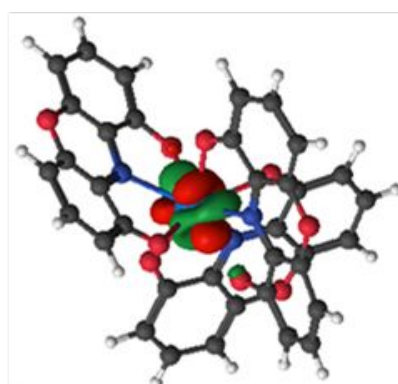

(0.03)

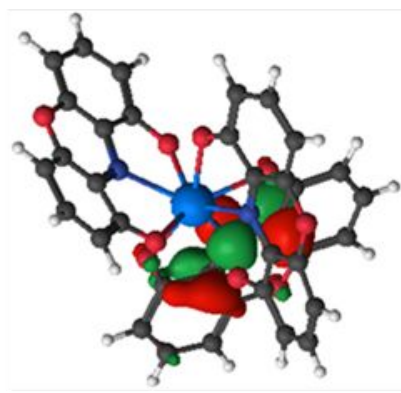

(0.06)

Figure S9: Active space orbitals of triplet spin state of $\mathrm{Np}\left(\mathrm{DOPO}^{q}\right)_{2}\left(\mathrm{DOPO}^{\mathrm{sq}}\right)$ (occupation numbers are in brackets). 


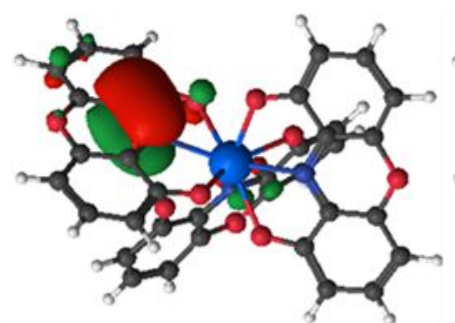

(1.94)

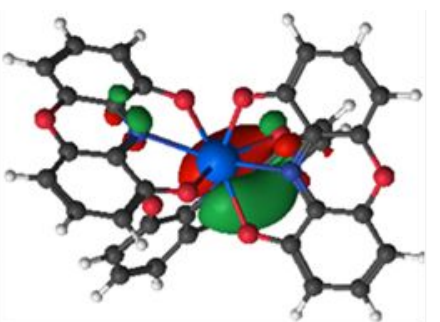

(1.94)

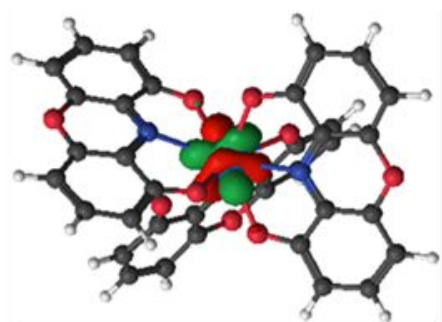

(0.10)

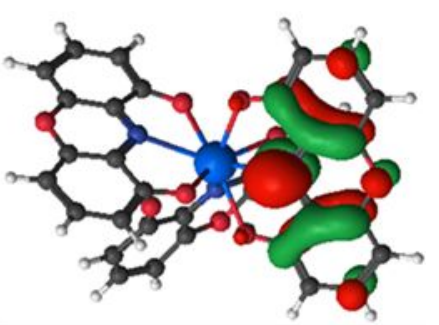

(1.00)

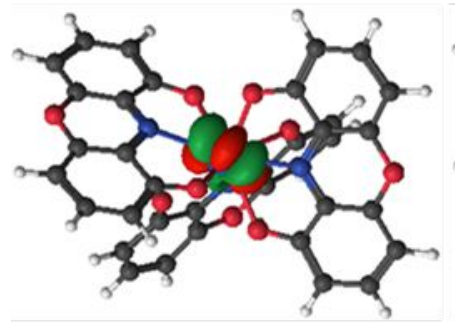

(0.12)

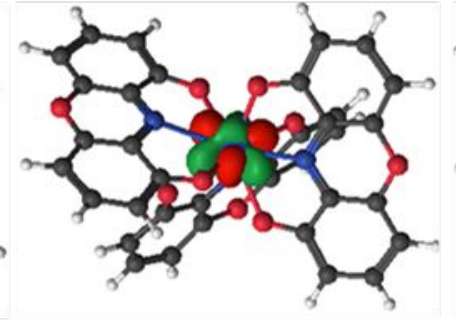

(0.95)

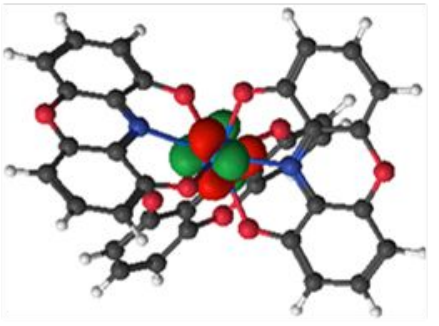

(0.88)

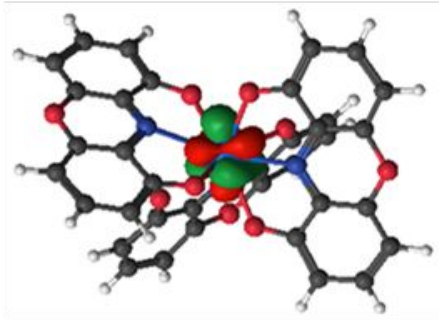

$(0.90)$

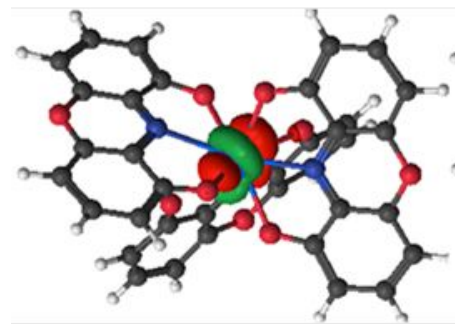

(0.05)

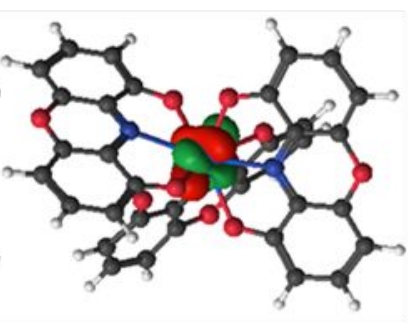

(1.00)

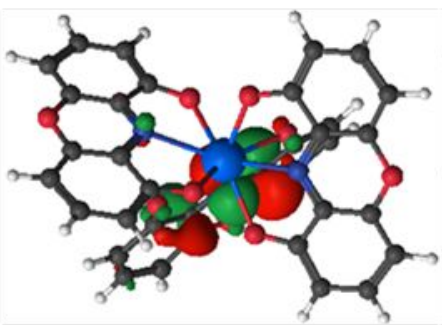

(0.06)

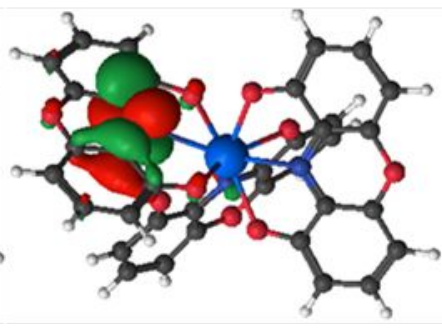

(0.06)

Figure S10: Active space orbitals of sextet spin state of $\mathrm{Pu}\left(\mathrm{DOPO}^{\mathrm{q}}\right)_{2}\left(\mathrm{DOPO}^{\mathrm{sq}}\right)$ (occupation numbers are in brackets). 


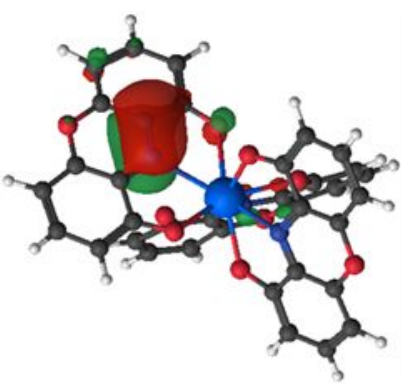

(1.94)

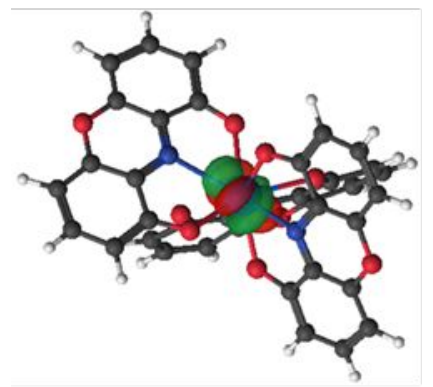

(0.11)

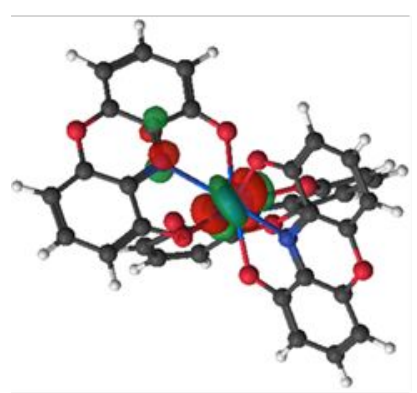

(0.06)

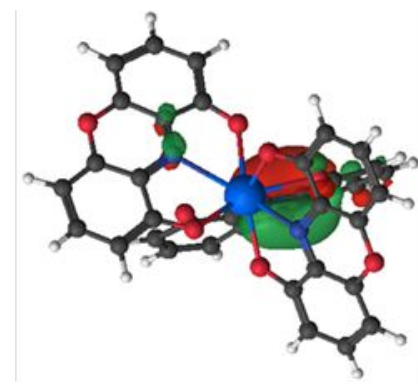

(1.94)

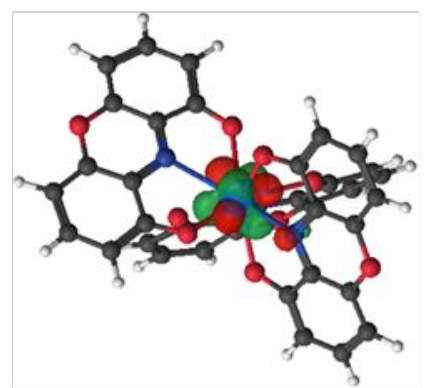

(0.94)

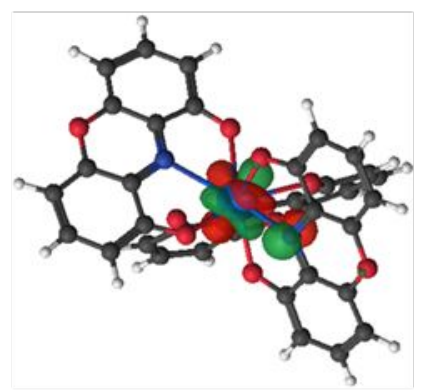

(0.98)

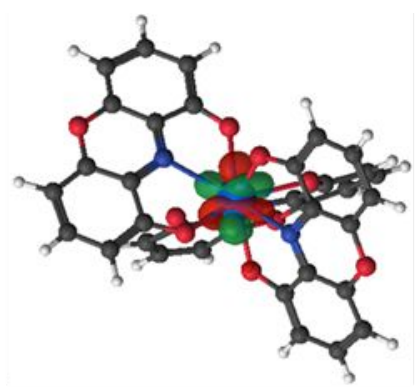

(0.10)

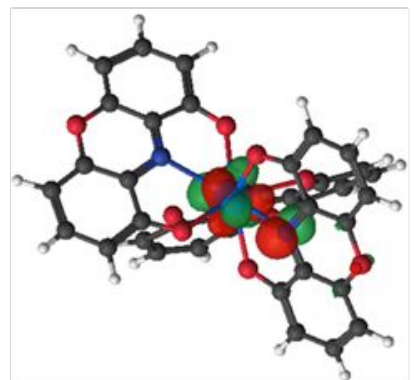

(0.86)

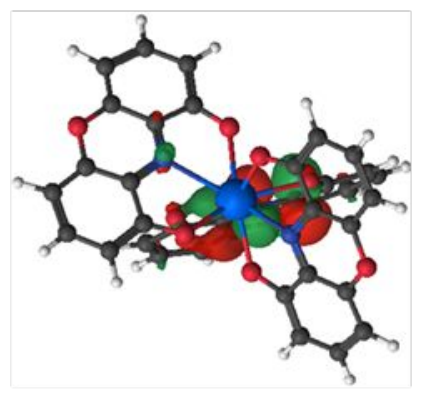

(0.06)

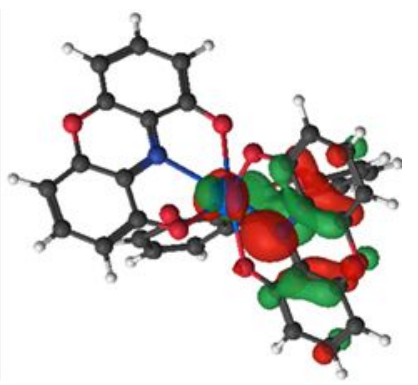

(1.05)

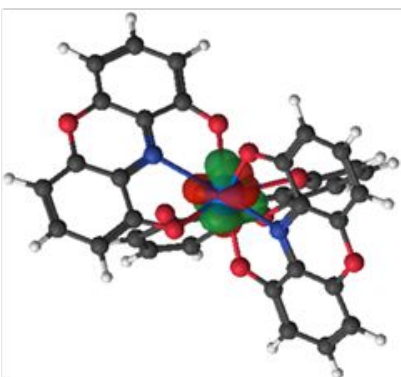

(0.90)

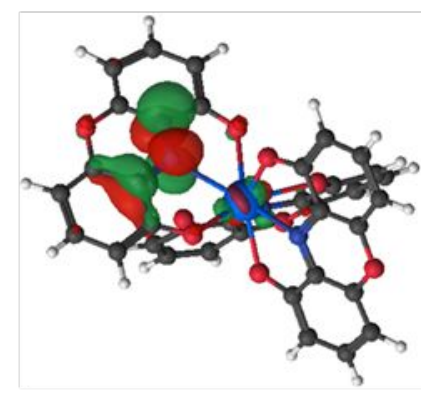

(0.06)

Figure S11: Active space orbitals of quartet spin state of $\mathrm{Pu}\left(\mathrm{DOPO}^{\mathrm{q}}\right)_{2}\left(\mathrm{DOPO}^{\mathrm{sq}}\right)$ (occupation numbers are in brackets). 


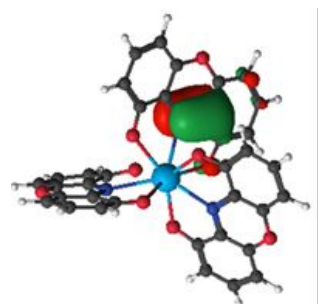

(1.93)

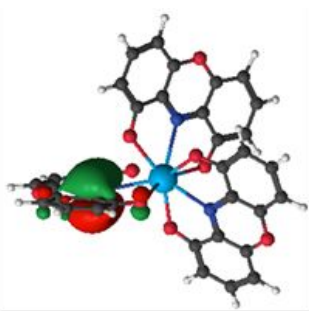

(1.94)

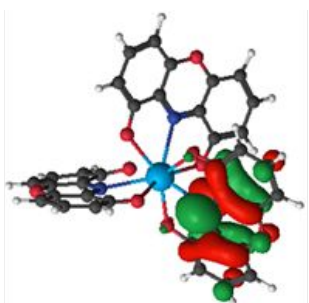

$(1.00)$

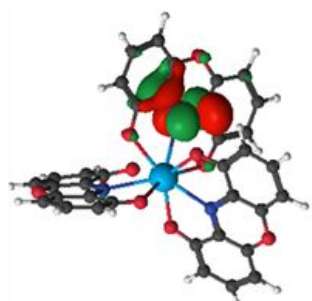

$(0.07)$

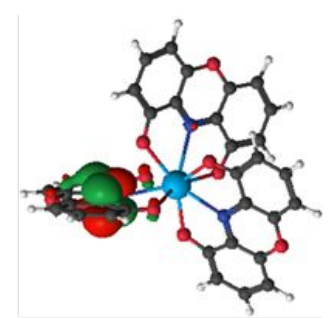

$(0.06)$

Figure S12: Active space orbitals of doublet spin state of $\mathrm{Th}\left(\mathrm{DOPO}^{\mathrm{q}}\right)_{2}\left(\mathrm{DOPO}^{\mathrm{sq}}\right)$ (occupation numbers are in brackets).

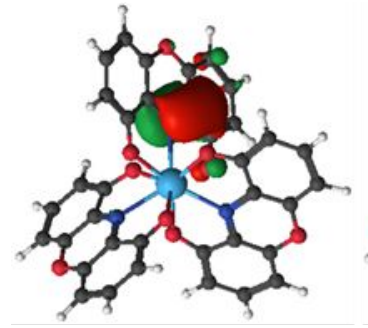

(1.94)

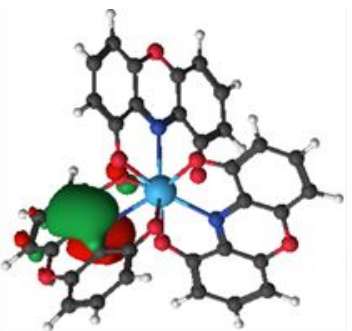

(1.94)

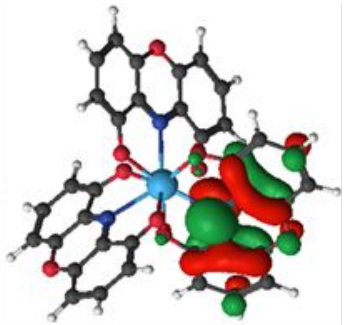

$(1.00)$

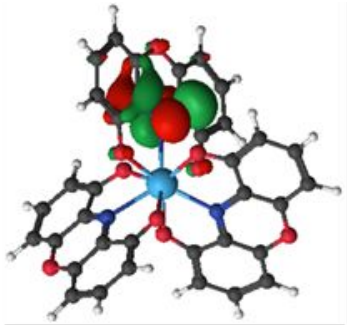

$(0.06)$

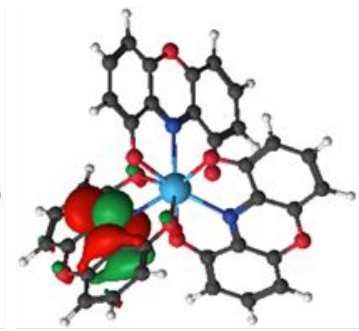

$(0.06)$

Figure S13: Active space orbitals of doublet spin state of $\mathrm{Hf}\left(\mathrm{DOPO}^{\mathrm{q}}\right)_{2}\left(\mathrm{DOPO}^{\mathrm{sq}}\right)$ (occupation numbers are in brackets). 


\section{XYZ optimized geometries}

\begin{tabular}{|c|c|c|c|}
\hline \multicolumn{4}{|c|}{$\begin{array}{l}\mathrm{U}\left(\mathrm{DOPO}^{\mathrm{q}}\right) 2\left(\mathrm{DOPO}^{\mathrm{sq}}\right) \\
67\end{array}$} \\
\hline \multicolumn{4}{|c|}{ SCF -17.29465172 a.u. } \\
\hline $\mathrm{C}$ & 5.791480 & 20.319370 & -39.679460 \\
\hline $\mathrm{C}$ & 6.698470 & 19.418670 & -40.278060 \\
\hline $\mathrm{C}$ & 7.898650 & 19.961280 & -40.706800 \\
\hline $\mathrm{C}$ & 8.328450 & 21.320940 & -40.574830 \\
\hline $\mathrm{C}$ & 7.437120 & 22.162960 & -39.926330 \\
\hline $\mathrm{C}$ & 6.206880 & 21.652180 & -39.475950 \\
\hline $\mathrm{C}$ & 4.378570 & 24.467580 & -38.359440 \\
\hline $\mathrm{C}$ & 4.472160 & 25.869960 & -38.293310 \\
\hline $\mathrm{C}$ & 5.720010 & 26.407990 & -38.617440 \\
\hline $\mathrm{C}$ & 6.852500 & 25.707660 & -39.066420 \\
\hline $\mathrm{C}$ & 6.694290 & 24.336340 & -39.203640 \\
\hline $\mathrm{C}$ & 5.488960 & 23.733020 & -38.816390 \\
\hline $\mathrm{C}$ & 2.946200 & 20.601560 & -35.607420 \\
\hline $\mathrm{C}$ & 3.352540 & 19.870850 & -34.473420 \\
\hline $\mathrm{C}$ & 2.333110 & 19.561610 & -33.586610 \\
\hline $\mathrm{C}$ & 0.958900 & 19.8 & 3610 \\
\hline $\mathrm{C}$ & 0.585920 & 20.5 & -34 \\
\hline $\mathrm{C}$ & 1.567830 & 20.9 & -35.7 \\
\hline $\mathrm{C}$ & -0.192610 & 22.352310 & -38.467490 \\
\hline $\mathrm{C}$ & -1.496730 & 22.719240 & -38.839630 \\
\hline $\mathrm{C}$ & -2.492840 & 22.437740 & -37.903000 \\
\hline $\mathrm{C}$ & -2.358630 & 21.8 & -36.633390 \\
\hline $\mathrm{C}$ & -1.068430 & & -36.249030 \\
\hline $\mathrm{C}$ & 0.000010 & 21.8 & -37 \\
\hline $\mathrm{C}$ & 3.056720 & 22.1 & -41 \\
\hline $\mathrm{C}$ & 3.240400 & 22.747700 & -43.177800 \\
\hline $\mathrm{C}$ & 2.747810 & 22.094020 & -44.268700 \\
\hline $\mathrm{C}$ & 2.107040 & 20.819160 & -44.320950 \\
\hline $\mathrm{C}$ & 2.043790 & 20.175360 & -43.113100 \\
\hline $\mathrm{C}$ & 2.450990 & 20.827060 & -41.934760 \\
\hline $\mathrm{C}$ & 1.45 & 18.7 & -39.267490 \\
\hline $\mathrm{C}$ & 0.91 & 17. & -39 \\
\hline $\mathrm{C}$ & 0.694510 & 16.675030 & 0200 \\
\hline $\mathrm{C}$ & 0.907850 & 17.023520 & -41.537160 \\
\hline $\mathrm{C}$ & 1.342610 & 18.326910 & -41.724260 \\
\hline $\mathrm{C}$ & 1.641840 & 19.122590 & -40.631640 \\
\hline $\mathrm{H}$ & 8.501510 & 19.366670 & -41.135930 \\
\hline $\mathrm{H}$ & 5.811590 & 27.349190 & -38.525280 \\
\hline $\mathrm{H}$ & 2.598180 & 19.063130 & -32.822400 \\
\hline $\mathrm{H}$ & -3.374080 & 22.6 & -38.145750 \\
\hline $\mathrm{H}$ & 2.844790 & 22.544540 & -45.099640 \\
\hline $\mathrm{H}$ & 0.353980 & 15.799630 & -40.043530 \\
\hline $\mathrm{N}$ & 5.259960 & 22.407440 & -38.904050 \\
\hline $\mathrm{N}$ & 1.267160 & 21.595130 & -36.837780 \\
\hline $\mathrm{N}$ & 2.222830 & 20.346300 & -40.727760 \\
\hline $\mathrm{O}$ & 4.550920 & 20.040950 & -39.305330 \\
\hline $\mathrm{O}$ & 7.706280 & 23.516080 & -39.722870 \\
\hline $\mathrm{O}$ & 3.284550 & 23.724540 & -38.102910 \\
\hline $\mathrm{O}$ & 3.720640 & 20.967310 & -36.594320 \\
\hline $\mathrm{O}$ & -0.731220 & 20.947920 & -35.028420 \\
\hline $\mathrm{O}$ & & $22.42 / 800$ & -39.2 \\
\hline
\end{tabular}




$\begin{array}{lrrr}\mathrm{O} & 3.365880 & 22.589510 & -40.765080 \\ \mathrm{O} & 1.499170 & 18.894580 & -42.990390 \\ \mathrm{O} & 1.848100 & 19.571040 & -38.358450 \\ \mathrm{U} & 2.985490 & 21.576960 & -38.693640 \\ \mathrm{H} & 0.256076 & 19.560104 & -32.888902 \\ \mathrm{H} & -3.219672 & 21.614944 & -36.000904 \\ \mathrm{H} & 4.381137 & 19.556482 & -34.322518 \\ \mathrm{H} & -1.718201 & 23.166610 & -39.804896 \\ \mathrm{H} & 3.637431 & 26.487596 & -37.973022 \\ \mathrm{H} & 7.779256 & 26.212291 & -39.326109 \\ \mathrm{H} & 9.287422 & 21.660450 & -40.955923 \\ \mathrm{H} & 6.441252 & 18.375463 & -40.442347 \\ \mathrm{H} & 3.705036 & 23.728323 & -43.249994 \\ \mathrm{H} & 1.758596 & 20.381328 & -45.251955 \\ \mathrm{H} & 0.682051 & 16.349857 & -42.358805 \\ \mathrm{H} & 0.723568 & 17.042757 & -38.050924\end{array}$

\section{$\mathrm{Np}$ (DOPOq)2(DOPOsq)}

\begin{tabular}{|c|c|c|c|}
\hline \\
\hline $\mathrm{O}$ & \multicolumn{3}{|c|}{67} \\
\hline & 18 & 10.449698 & 4.754078 \\
\hline $\mathrm{Np}$ & 9.983999 & 12.207041 & 4.061814 \\
\hline $\mathrm{O}$ & 5.974910 & 13.607497 & 7.044104 \\
\hline $\mathrm{O}$ & 9.622849 & 14.460788 & 4.142159 \\
\hline $\mathrm{O}$ & 8.624088 & 12.061297 & 2.138858 \\
\hline $\mathrm{O}$ & 12.266521 & 13.017357 & 4.124261 \\
\hline $\mathrm{O}$ & 10.366075 & 12.671679 & 6.384590 \\
\hline $\mathrm{O}$ & 12.719529 & 8.610763 & 6.898646 \\
\hline $\mathrm{O}$ & 10.970813 & 10.346930 & 2.856256 \\
\hline $\mathrm{N}$ & 7.955165 & 12.855418 & 5.198071 \\
\hline $\mathrm{N}$ & 11.085067 & 12.747978 & 1.822429 \\
\hline $\mathrm{N}$ & 11.358067 & 10.510426 & 5.401027 \\
\hline $\mathrm{C}$ & 7.668750 & 10.592952 & 5.605126 \\
\hline $\mathrm{C}$ & 6.971848 & 9.546580 & 6.263882 \\
\hline $\mathrm{C}$ & 6.040150 & 9.900042 & 7.223455 \\
\hline $\mathrm{H}$ & 5.623459 & 9.211882 & 7.691587 \\
\hline $\mathrm{C}$ & 5.664517 & 11.267176 & 7.562354 \\
\hline $\mathrm{C}$ & 6.278190 & 12.234135 & 6.827443 \\
\hline $\mathrm{C}$ & 7.205368 & 11.918043 & 5.875482 \\
\hline $\mathrm{C}$ & 8.677225 & 15.027572 & 4.910198 \\
\hline $\mathrm{C}$ & 6.781878 & 14.576014 & 6.431427 \\
\hline $\mathrm{C}$ & 7.759356 & 14.171167 & 5.521354 \\
\hline $\mathrm{C}$ & 9.074144 & 11.989670 & 0.944344 \\
\hline $\mathrm{C}$ & 10.451697 & 12.400744 & 0.727294 \\
\hline $\mathrm{C}$ & 13.009404 & 13.177737 & 3.114805 \\
\hline $\mathrm{C}$ & 14.383706 & 13.495386 & 3.149077 \\
\hline $\mathrm{C}$ & 14.479416 & 13.222894 & 0.613058 \\
\hline $\mathrm{C}$ & 13.169480 & 12.964415 & 0.571174 \\
\hline $\mathrm{C}$ & 12.390421 & 12.987771 & 1.797295 \\
\hline $\mathrm{C}$ & 11.026928 & 11.936728 & 7.181571 \\
\hline $\mathrm{C}$ & 11.317749 & 12.268391 & 8.563811 \\
\hline $\mathrm{C}$ & 12.110813 & 11.362159 & 9.237796 \\
\hline $\mathrm{H}$ & 12.321112 & 11.581679 & 10.117597 \\
\hline $\mathrm{C}$ & 12.649642 & 10.125822 & 8.758012 \\
\hline $\mathrm{C}$ & 12.302532 & 9.780145 & 7.497619 \\
\hline $\mathrm{C}$ & 11.561451 & 10.715962 & 6.697974 \\
\hline
\end{tabular}




$\begin{array}{cccc}\mathrm{C} & 11.573714 & 9.341043 & 3.396586 \\ \mathrm{C} & 11.993793 & 8.165432 & 2.711176 \\ \mathrm{C} & 12.500021 & 7.145532 & 3.503205 \\ \mathrm{H} & 12.721704 & 6.356175 & 3.066484 \\ \mathrm{C} & 12.720210 & 7.170445 & 4.915913 \\ \mathrm{C} & 12.432425 & 8.381869 & 5.551817 \\ \mathrm{C} & 11.818502 & 9.400212 & 4.820715 \\ \mathrm{C} & 8.596969 & 16.394707 & 5.220535 \\ \mathrm{C} & 6.606751 & 15.916677 & 6.663702 \\ \mathrm{C} & 8.351093 & 11.496070 & -0.194199 \\ \mathrm{C} & 11.078213 & 12.301090 & -0.536903 \\ \mathrm{C} & 15.027093 & 13.506286 & 1.919147 \\ \mathrm{O} & 12.443810 & 12.682579 & -0.583359 \\ \mathrm{C} & 7.563922 & 16.777752 & 6.058261 \\ \mathrm{C} & 9.059378 & 11.474270 & -1.382244 \\ \mathrm{C} & 10.401011 & 11.858874 & -1.629753 \\ \mathrm{H} & 15.931422 & 13.723578 & 1.939937 \\ \mathrm{H} & 7.487398 & 17.686663 & 6.241035 \\ \mathrm{H} & 8.588868 & 11.163193 & -2.120731 \\ \mathrm{H} & 13.162263 & 6.327731 & 5.440670 \\ \mathrm{H} & 11.848397 & 8.055423 & 1.639794 \\ \mathrm{H} & 13.250458 & 9.488607 & 9.402081 \\ \mathrm{H} & 10.851575 & 11.772626 & -2.614249 \\ \mathrm{H} & 7.321460 & 11.159329 & -0.113542 \\ \mathrm{H} & 14.903297 & 13.693498 & 4.082844 \\ \mathrm{H} & 10.956416 & 13.189526 & 9.012787 \\ \mathrm{H} & 4.934496 & 11.482991 & 8.337097 \\ \mathrm{H} & 9.302210 & 17.111133 & 4.806454 \\ \mathrm{H} & 5.841635 & 16.298101 & 7.334753 \\ \mathrm{H} & 7.226860 & 8.505677 & 6.075087 \\ \mathrm{H} & 15.116490 & 13.233624 & -0.267559\end{array}$

$\mathrm{Pu}\left(\mathrm{DOPO}^{\mathrm{q}}\right) 2\left(\mathrm{DOPO}^{\mathrm{sq}}\right)$ 67

SCF -17.23156824 a.u.

$\begin{array}{llll}\text { C } & 41.997660 & 14.606600 & 7.874390\end{array}$

$\begin{array}{llll}\text { C } & 42.614370 & 13.401020 & 8.246570\end{array}$

$\begin{array}{llll}\text { C } & 43.228970 & 13.393690 & 9.495750\end{array}$

$\begin{array}{llll}\text { C } & 43.276390 & 14.451700 & 10.435670\end{array}$

$\begin{array}{llll}\text { C } & 42.600250 & 15.602850 & 10.072070\end{array}$

$\begin{array}{llll}\text { C } & 41.962370 & 15.664010 & 8.826540\end{array}$

$\begin{array}{llll}\text { C } & 40.899340 & 19.047260 & 8.480830\end{array}$

$\begin{array}{llll}\text { C } & 40.962620 & 20.260060 & 9.174290\end{array}$

$\begin{array}{llll}\text { C } & 41.487160 & 20.214660 & 10.463380\end{array}$

$\begin{array}{llll}\text { C } & 41.998360 & 19.084370 & 11.136660\end{array}$

$\begin{array}{llll}\text { C } & 41.989960 & 17.911060 & 10.405350\end{array}$

$\begin{array}{llll}\text { C } & 41.410970 & 17.888230 & 9.129410\end{array}$

$\begin{array}{llll}\text { C } & 37.641150 & 16.187470 & 5.998860\end{array}$

$\begin{array}{llll}\text { C } & 36.479460 & 15.398940 & 6.183790\end{array}$

$\begin{array}{llll}\text { C } & 35.461780 & 15.591570 & 5.270110\end{array}$

$\begin{array}{llll}\text { C } & 35.429420 & 16.464290 & 4.147260\end{array}$

$\begin{array}{llll}\text { C } & 36.567830 & 17.206890 & 3.958300\end{array}$

$\begin{array}{llll}\text { C } & 37.639980 & 17.092630 & 4.880560\end{array}$

$\begin{array}{llll}\text { C } & 40.321230 & 19.005970 & 3.501730\end{array}$

$\begin{array}{llll}\text { C } & 40.569970 & 19.880290 & 2.405890\end{array}$

$\begin{array}{llll}\text { C } & 39.526600 & 20.065420 & 1.538130\end{array}$ 


\begin{tabular}{|c|c|c|c|}
\hline $\mathrm{C}$ & 38.214980 & 19.483050 & 1.565280 \\
\hline $\mathrm{C}$ & 37.945210 & 18.705440 & 2.660080 \\
\hline $\mathrm{C}$ & 38.964130 & 18.503010 & 3.641820 \\
\hline $\mathrm{C}$ & 44.058330 & 17.335000 & 5.820030 \\
\hline $\mathrm{C}$ & 45.386890 & 17.793000 & 6.044020 \\
\hline $\mathrm{C}$ & 46.355280 & 17.343610 & 5.175740 \\
\hline $\mathrm{C}$ & 46.201210 & 16.440450 & 4.078030 \\
\hline $\mathrm{C}$ & 44.941090 & 15.924760 & 3.925430 \\
\hline $\mathrm{C}$ & 43.881030 & 16.393350 & 4.738790 \\
\hline $\mathrm{C}$ & 40.915660 & 14.968810 & 3.383900 \\
\hline $\mathrm{C}$ & 40.552010 & 14.039790 & 2.363840 \\
\hline $\mathrm{C}$ & 41.583450 & 13.398010 & 1.713020 \\
\hline $\mathrm{C}$ & 42.984230 & 13.590330 & 1.882540 \\
\hline $\mathrm{C}$ & 43.324290 & 14.586520 & 2.765570 \\
\hline $\mathrm{C}$ & 42.323930 & 15.221890 & 3.531840 \\
\hline $\mathrm{H}$ & 43.665850 & 12.587990 & 9.745690 \\
\hline $\mathrm{H}$ & 41.503080 & 21.034900 & 10.942390 \\
\hline $\mathrm{H}$ & 34.679000 & 15.071960 & 5.410310 \\
\hline $\mathrm{H}$ & 39.694160 & 20.662200 & 0.818250 \\
\hline $\mathrm{H}$ & 47.233570 & 17.673950 & 5.323690 \\
\hline $\mathrm{H}$ & 41.328130 & 12.745900 & 1.070980 \\
\hline $\mathrm{N}$ & 41.347930 & 16.773190 & 8.378190 \\
\hline $\mathrm{N}$ & 38.778130 & 17.761800 & 4.713120 \\
\hline $\mathrm{N}$ & 42.618870 & 16.075560 & 4.510440 \\
\hline $\mathrm{O}$ & 41.449960 & 14.868010 & 6.706140 \\
\hline $\mathrm{O}$ & 42.539100 & 16.722970 & 10.903600 \\
\hline $\mathrm{O}$ & 40.428030 & 18.858330 & 7.257760 \\
\hline $\mathrm{O}$ & 38.707200 & 5030 & 6.717070 \\
\hline $\mathrm{O}$ & 36.726600 & 18.085080 & 2.889160 \\
\hline $\mathrm{O}$ & 41.185210 & 18.619450 & 4.351660 \\
\hline $\mathrm{O}$ & 43.024570 & 17.692840 & 6.462160 \\
\hline $\mathrm{O}$ & 44.640500 & 14.978840 & 2.957470 \\
\hline $\mathrm{O}$ & 40.114330 & 15.589660 & 4.153380 \\
\hline $\mathrm{Pu}$ & 40.793820 & 16.956830 & 6.037740 \\
\hline $\mathrm{H}$ & 42.412990 & 19.1 & 12.139096 \\
\hline $\mathrm{H}$ & 34.570748 & 16.5 & 3.483632 \\
\hline $\mathrm{H}$ & 37.485965 & 19.686135 & 0.785893 \\
\hline $\mathrm{H}$ & 41.540400 & 20.349595 & 2.266203 \\
\hline $\mathrm{H}$ & 40.603298 & 21.186397 & 8.732675 \\
\hline $\mathrm{H}$ & 43.795944 & 14.354968 & 11.385157 \\
\hline $\mathrm{H}$ & 42.648847 & 12.545322 & 7.577397 \\
\hline $\mathrm{H}$ & 36.402091 & 14.686481 & 7.000931 \\
\hline $\mathrm{H}$ & 45.609533 & 18.492432 & 6.846238 \\
\hline $\mathrm{H}$ & 47.0 & 16.1 & 3.454649 \\
\hline $\mathrm{H}$ & 43.717625 & 13.043811 & 1.295816 \\
\hline $\mathrm{H}$ & 39.508773 & 13.807792 & 2.163400 \\
\hline \multicolumn{4}{|c|}{$\mathrm{Hf}\left(\mathrm{DOPO}^{\mathrm{q}}\right) 2\left(\mathrm{DOPO}^{\mathrm{sq}}\right)$} \\
\hline \multicolumn{4}{|c|}{ SCF -17.23193337 a.u. } \\
\hline $\mathrm{C}$ & 16.102030 & 20.060190 & 24.488990 \\
\hline $\mathrm{C}$ & 16.283910 & 19.738110 & 25.879690 \\
\hline $\mathrm{C}$ & 15.401320 & 18.765560 & 26.427300 \\
\hline $\mathrm{C}$ & 14.512700 & 18.188070 & 25.527470 \\
\hline $\mathrm{C}$ & 14.365770 & 18.434700 & 24.114730 \\
\hline $\mathrm{C}$ & 630 & 19.417010 & \\
\hline
\end{tabular}




\begin{tabular}{|c|c|c|c|}
\hline & 30 & 80 & \\
\hline & 17.998150 & 22.294740 & 201815 \\
\hline & 18.065190 & 22.743620 & \\
\hline & 7.178680 & 179480 & \\
\hline & 5.185440 & 21.163850 & \\
\hline & 5.086770 & 20.770970 & \\
\hline & 570 & 32890 & \\
\hline & 0.074810 & 19.065820 & \\
\hline & 0.595020 & & \\
\hline & 50 & $17.2^{\prime}$ & \\
\hline & 00 & 220 & \\
\hline & & & \\
\hline & 740 & & \\
\hline & 000 & 5390 & \\
\hline & 20 & & \\
\hline & 090 & 370 & \\
\hline$c$ & 50 & & \\
\hline $\mathrm{C}$ & 21. & & \\
\hline 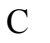 & 50 & & \\
\hline & 20 & 30 & \\
\hline & 80 & 310 & \\
\hline & & & \\
\hline & & 27. & \\
\hline & & & \\
\hline $\mathrm{C}$ & & & \\
\hline $\mathrm{C}$ & & & \\
\hline$\therefore$ & 0 & & \\
\hline 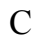 & 0 & & \\
\hline 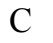 & 0 & & \\
\hline & & & \\
\hline & 13. & & \\
\hline $\mathrm{H}$ & & & \\
\hline $\mathrm{H}$ & & & \\
\hline & & & \\
\hline & & & \\
\hline & $4 .(1$ & & \\
\hline $\mathrm{Hf}$ & & & \\
\hline $\mathrm{N}$ & & & \\
\hline $\mathrm{N}$ & & & \\
\hline $\mathrm{N}$ & & & \\
\hline $\mathrm{O}$ & & & \\
\hline $\mathrm{O}$ & & & \\
\hline $\mathrm{O}$ & & & \\
\hline $\mathrm{O}$ & 20 & 19. & \\
\hline O & 220 & & \\
\hline O & 19 & & \\
\hline $\mathrm{O}$ & & & \\
\hline $\mathrm{O}$ & & & \\
\hline $\mathrm{O}$ & & & \\
\hline 1 & & & \\
\hline & 58 & & \\
\hline & & & \\
\hline I & & & \\
\hline$\Pi$ & 15.553450 & 20.765659 & \\
\hline $\mathrm{H}$ & 18.798332 & 23.485653 & 20.02 \\
\hline & 23.538262 & 22.651415 & 4.57 \\
\hline
\end{tabular}




$\begin{array}{cccc}\mathrm{H} & 22.709632 & 26.925420 & 25.006774 \\ \mathrm{H} & 23.000518 & 16.759839 & 25.879486 \\ \mathrm{H} & 20.260022 & 17.894969 & 22.671465 \\ \mathrm{H} & 20.069360 & 23.022879 & 29.969167 \\ \mathrm{H} & 22.799380 & 19.602752 & 30.112187\end{array}$

\begin{tabular}{|c|c|c|c|}
\hline \multicolumn{4}{|c|}{$\begin{array}{l}\mathrm{Th}\left(\mathrm{DOPO}^{\mathrm{q}}\right) 2\left(\mathrm{DOPO}^{\mathrm{sq}}\right) \\
67\end{array}$} \\
\hline \multicolumn{4}{|c|}{ SCF -17.28253380 a.u. } \\
\hline $\mathrm{C}$ & 12.504440 & 24.465470 & -15.024350 \\
\hline $\mathrm{C}$ & 11.571850 & 25.088650 & -14.141970 \\
\hline $\mathrm{C}$ & 12.083250 & 26.096800 & -13.336900 \\
\hline $\mathrm{C}$ & 13.400250 & 26.576590 & -13.247720 \\
\hline $\mathrm{C}$ & 14.319420 & 25.919860 & -14.067090 \\
\hline $\mathrm{C}$ & 13.851030 & 24.918740 & -14.961870 \\
\hline $\mathrm{C}$ & 16.823500 & 23.716840 & -16.594100 \\
\hline $\mathrm{C}$ & 18.218010 & 23.960100 & -16.567940 \\
\hline $\mathrm{C}$ & 18.660980 & 24.889020 & -15.635650 \\
\hline $\mathrm{C}$ & 17.881260 & 25.658920 & \\
\hline $\mathrm{C}$ & 16. & 25.4 & \\
\hline $\mathrm{C}$ & 16. & 24. & \\
\hline $\mathrm{C}$ & 15.255730 & 20.839 & 400 \\
\hline $\mathrm{C}$ & 15.682690 & 20.714280 & -12.953800 \\
\hline $\mathrm{C}$ & 16.592610 & 19.690770 & \\
\hline $\mathrm{C}$ & & 18.789240 & -13 \\
\hline $\mathrm{C}$ & & & \\
\hline $\mathrm{C}$ & & & \\
\hline $\mathrm{C}$ & & $19 .^{\circ}$ & \\
\hline $\mathrm{C}$ & 16 & 19. & \\
\hline $\mathrm{C}$ & 17. & $18.1^{\prime}$ & 550 \\
\hline $\mathrm{C}$ & 17.514770 & 17.769550 & 350 \\
\hline $\mathrm{C}$ & 16 & 18.442550 & 300 \\
\hline $\mathrm{C}$ & & 19.404 & \\
\hline $\mathrm{C}$ & 10. & 20.6 & 110 \\
\hline $\mathrm{C}$ & & 20.0 & \\
\hline $\mathrm{C}$ & 8.4 & 20.4 & \\
\hline $\mathrm{C}$ & 8.12 & 21.366270 & 10 \\
\hline $\mathrm{C}$ & 9.191230 & 21.969960 & -18 . \\
\hline $\mathrm{C}$ & 10.523510 & 21.678930 & 920 \\
\hline $\mathrm{C}$ & 12.6 & 23.931200 & 650 \\
\hline $\mathrm{C}$ & 12. & 24.75 & 5270 \\
\hline $\mathrm{C}$ & 11. & 24.925150 & -21.299030 \\
\hline $\mathrm{C}$ & & 24.387250 & \\
\hline $\mathrm{C}$ & & 23.5 & \\
\hline $\mathrm{C}$ & 11.389620 & 23.26 & 10 \\
\hline $\mathrm{H}$ & 11.455370 & 26.522330 & 65720 \\
\hline $\mathrm{H}$ & 19.599940 & 25.0318 & 5490 \\
\hline $\mathrm{H}$ & 16.855360 & 19.586 & 100 \\
\hline $\mathrm{H}$ & 17.458430 & 17.73 & 9810 \\
\hline $\mathrm{H}$ & 7.774440 & 19.950890 & 400 \\
\hline $\mathrm{H}$ & 11.2 & 25.47 & 2820 \\
\hline $\mathrm{N}$ & 14.706090 & 24.2896 & -15.785800 \\
\hline $\mathrm{N}$ & 15.356790 & 20.11911 & -16.511300 \\
\hline $\mathrm{N}$ & 11.560120 & 22.34851 & -18.253890 \\
\hline $\mathrm{O}$ & & 23.52695 & \\
\hline $\mathrm{O}$ & 15.654150 & 26.213570 & -14.028840 \\
\hline
\end{tabular}




$\begin{array}{cccc}\mathrm{O} & 16.224190 & 22.828180 & -17.298270 \\ \mathrm{O} & 14.423890 & 21.744740 & -14.767600 \\ \mathrm{O} & 17.290870 & 18.172170 & -15.953470 \\ \mathrm{O} & 14.579610 & 20.745080 & -18.861610 \\ \mathrm{O} & 12.079780 & 20.512620 & -16.475990 \\ \mathrm{O} & 9.022430 & 22.899600 & -19.200650 \\ \mathrm{O} & 13.667770 & 23.747750 & -18.896390 \\ \mathrm{Th} & 13.894170 & 22.105940 & -17.043670 \\ \mathrm{H} & 18.273829 & 17.005887 & -18.202154 \\ \mathrm{H} & 17.892260 & 18.035327 & -13.320319 \\ \mathrm{H} & 15.860035 & 19.412958 & -20.926663 \\ \mathrm{H} & 15.302715 & 21.370965 & -12.175274 \\ \mathrm{H} & 18.902471 & 23.411291 & -17.209855 \\ \mathrm{H} & 18.344431 & 26.354468 & -14.020656 \\ \mathrm{H} & 13.689643 & 27.362736 & -12.555677 \\ \mathrm{H} & 10.526461 & 24.793331 & -14.118626 \\ \mathrm{H} & 9.070552 & 24.611340 & -21.327044 \\ \mathrm{H} & 7.086815 & 21.556629 & -17.822891 \\ \mathrm{H} & 9.900964 & 19.285730 & -15.353968 \\ \mathrm{H} & 13.390967 & 25.241005 & -21.178897\end{array}$

\section{References}

[1] Nonius (1998). Collect Users Manual, Nonius Delft, The Netherlands.

[2] Otwinowski Z, Minor W., Processing of X-ray diffraction data collected in oscillation mode.

Methods Enzymol. 1997, 276, 307-326.

[3] a) SHELXTL suite of programs, Version 6.14, 2000-2003, Bruker Advanced X-ray Solutions, Bruker AXS Inc., Madison, Wisconsin: USA)

[4] Sheldrick GM. A short history of SHELX. Acta Cryst. A. 2008, 64, 112-122.

[5] Hübschle CB, Sheldrick GM, Dittrich B. ShelXle: a Qt graphical user interface for SHELXL. J. Appl. Crystallogr. 2011, 44, 1281-1284.

[6] a) Sheldrick GM. University of Göttingen, Germany, 2014. b) Sheldrick GM. Crystal structure refinement with SHELXL. Acta Cryst C 2015, 71, 3-8. 